\title{
ON THE TOPOLOGY OF CYCLIC PRODUCTS OF SPHERES
}

\author{
BY \\ S. D. LIAO \\ INTRODUCTION
}

We propose to study certain topological properties of cyclic products of spheres. Applications may be made in the theory of fiber bundles $\left.{ }^{1}\right)$. Our success is as follows.

We shall introduce first the notion of "regular imbedding of a space into its $\Gamma$-products" (this notion appears in a more general situation). Let $X$ be a locally compact, paracompact Hausdorff space. By the $\Gamma$-product $X^{\Gamma}$ of $X$ we mean the orbit space over a $q$-fold Cartesian product $X^{q}$ of $X$ on which a group $\Gamma$ of permutations of factors of $X^{q}$ acts. It is the $q$-fold cyclic product or $q$-fold symmetric product if $\Gamma$ is a cyclic group of order $q$ or the whole symmetric group. Let $s_{0} \in X$ be any given point. By identifying any $x \in X$ with $\bar{I}\left(x, s_{0}, s_{0}, \cdots, s_{0}\right) \in X^{\mathrm{r}}$ where $\bar{I}$ denotes the natural map: $X^{q} \rightarrow X^{\mathrm{r}}, X$ is topologically imbedded in $X^{\mathrm{T}}$. We call this imbedding a regular imbedding. Let $G$ be a coefficient group for cohomology groups. We show then that every cohomology class $v \in H^{s}(X, G)$ extends to a certain cohomology class $\mu(v)$ $\in H^{s}\left(X^{\mathbf{\Gamma}}, G\right)$ such that $\mu: H^{s}(X, G) \rightarrow H^{s}\left(X^{\mathbf{\Gamma}}, G\right)$ is an into-isomorphism and $\eta \mu$ is the identity isomorphism of $H^{s}(X, G)$ where $\eta: H^{s}\left(X^{\Gamma}, G\right) \rightarrow H^{s}(X, G)$ is the projection homormorphism.

In general, no more cohomology relations between $X$ and $X^{\mathbf{r}}$ are studied in this paper. We consider the $p$-fold cyclic product, to be denoted by $\vartheta_{n p}$ of an $n$-sphere $S_{n}$ with $p$ prime and $n \geqq 2 . \vartheta_{n p}$ has vanishing integral cohomology groups $H^{s}\left(\vartheta_{n p}, Z\right)$ of dimensions between 0 and $n$ and has infinite cyclic $n$-dimensional integral cohomology group $H^{n}\left(\vartheta_{n p}, Z\right)$. If $S_{n}$ is regularly imbedded in $\vartheta_{n p}$, the into-isomorphism $\mu$ above maps a generator of $H^{n}\left(S_{n}, Z\right)$ into a generator $g_{n}^{*}$ of $H^{n}\left(\vartheta_{n p}, Z\right)$. We shall calculate in $\vartheta_{n p}$ explicitly the iterated cyclic reduced powers mod $p$ of $g_{n}^{*}$. In particular, we have that $\mathcal{P}^{k} g_{n}^{*} \neq 0$ for all $2 k \leqq n$.

In the last chapter of this paper, we study certain homotopy properties of 2 -fold and 3-fold cyclic products of spheres. These are partial results on the problem of "symmetrization killing homotopy groups" as we state in

Presented to the Society, June 20, 1953; received by the editors July 30, 1953.

(1) For instance, in the author's paper, On the theory of obstructions of fiber bundles (Thesis, University of Chicago, 1952; to appear in the Ann. of Math.), a fiber bundle $B^{*}$ related to a given sphere bundle $B$ is constructed with fiber the 2 -fold cyclic product of a sphere to deduce the formula of secondary obstructions of $B$. The notion "regular imbedding" in $\$ 3$ and the results $\pi_{i}\left(S_{n} * S_{n}\right)=0$ etc. for $i=n+1, n+2$ in (13.3), (13.6) of $\$ 13$ are drawn from the author's thesis. 
$\$ 10$. We show that $\pi_{i}\left(\vartheta_{n 3}\right)$ vanishes for $i=n+1, n+2$, and the 2-primary subgroup of $\pi_{n+2}\left(\vartheta_{n 2}\right)$ vanishes for $n \geqq 5$. Let $S_{n}$ be regularly imbedded in $\vartheta_{n p}$. We show that every map $f: \partial E_{n+4} \rightarrow S_{n}$ which represents a 3-primary element $e_{f}$ of $\pi_{n+3}\left(S_{n}\right), n \geqq 3$, extends in $\vartheta_{n 3}$ over $E_{n+4}$ where $E_{n+4}$ denotes an oriented $(n+4)$-cell. Actually, more results on this homotopy property of $\vartheta_{n p}$ are obtained. For instance, we show that if $F: E_{n+4} \rightarrow_{n 3}$ is an extension of $f$ above, then $e_{f}$ is characterized by $F^{*} \xi^{-1} \mathcal{P}^{1} g_{n}^{*} \in H^{n+4}\left(E_{n+4}, \partial E_{n+4} ; Z_{3}\right)$ where $Z_{3}$ denotes the groups of integers $\bmod 3$ and $\xi$ is the injection homomorphism: $H^{n+4}\left(\vartheta_{n 3}, S_{n} ; Z_{3}\right) \rightarrow H^{n+4}\left(\vartheta_{n 3}, Z_{3}\right)$ which is an isomorphism. In the last section of this paper, we consider an $(n-1)$-connected finite complex $K, n \geqq 2$, and regularly imbed $K$ into its 2 -fold symmetric product. We show how this symmetric product is related to the problem of secondary obstructions of maps of a complex into $K$, a solution of which was originally given by N. E. Steenrod [10] and generalized by J. H. C. Whitehead [16].

\section{I. $\Gamma$-products. General properties}

1. $\Gamma$-products, homotopy type. Let $X$ be a locally compact, paracompact Hausdorff space, and $X^{q}$ the $q$-fold Cartesian product of $X$. Let $\Gamma$ be a group of permutations of the $q$ letters $1,2, \cdots, q$. We shall regard $\Gamma$ as a transformation group acting on $X^{q}$ in a natural fashion as follows: For any $\gamma \in \Gamma$ and $x=\left(x_{1}, x_{2}, \cdots, x_{q}\right) \in X^{q}$ we set $\gamma(x)=\left(x_{\gamma(1)}, x_{\gamma(2)}, \cdots, x_{\gamma(q)}\right)$. The orbit space over $X^{q}$ relative to $\Gamma$ (obtained by identifying any two points $x, x^{\prime}$ of $X^{q}$ into a single point whenever $x^{\prime}=\gamma(x)$ for some $\gamma \in \Gamma$ ) will be denoted by $X^{\Gamma} . X^{\Gamma}$ is a locally compact, paracompact Hausdorff space. It may be called the $\Gamma$-product of $X$. It is the $q$-fold cyclic product or the $q$-fold symmetric product of $X$ when $\Gamma$ is the cyclic group of order $q$ or the symmetric group $\Sigma_{q}$ of the $q$ letters $1,2, \cdots, q$. We shall write $\bar{I}$ for the identification map: $X^{q} \rightarrow X^{\mathbf{r}}$. Let $Y$ be also a locally compact, paracompact Hausdorff space and $f: X \rightarrow Y$ a map. We shall write $f^{q}: X^{q} \rightarrow Y^{q}$, and $f^{\Gamma}: X^{\Gamma} \rightarrow Y^{\Gamma}$ respectively for the $q$-fold Cartesian product of $f$ and the map determined naturally by $f$ such that $f^{\Gamma} \bar{I}=\bar{I} f^{q}$. For $q=2$, we write $X^{\Sigma_{2}}$ as $X * X$ and write $f^{\Sigma_{2}}$ as $f * f\left({ }^{2}\right)$.

We shall introduce a natural simplicial decomposition of the Cartesian product and hence of the $\Gamma$-product of a complex $\left({ }^{3}\right)$. The meaning of an ordered simplex and of an ordered simplicial complex will be understood as usual. Every simplicial complex can be ordered. We write an ordered $s$-simplex

(2) For known topological properties of cyclic and symmetric products, one may see [7, p. 184] and an announcement of S. K. B. Stein, Bull. Amer. Math. Soc. vol. 58 (1952) p. 207.

${ }^{(3)}$ The simplicial decomposition we shall introduce is simpler than that used by $\mathbf{M}$. Richardson, On the homology characters of symmetric products, Duke Math. J. vol. 1 (1935) pp. 50-69.

By a complex we mean one of which a locally finite simplicial decomposition exists. Later, we shall consider also the $C W$-complexes of J. H. C. Whitehead $[14$, p. 223], which we shall refer to as cellular complexes. 
$\sigma$ as $\sigma=\left[w_{0}<w_{1}<\cdots<w_{s}\right]$ with vertices $w_{0}, w_{1}, \cdots, w_{s}$ and order $<$. Let $\sigma_{k}=\left[w_{0 k}<w_{1 k}<\cdots<w_{s_{k}}\right]$ be given ordered simplexes, $k=1,2, \cdots, q$. A simplicial decomposition of $\sigma^{\not}=\sigma_{1} \times \sigma_{2} \times \cdots \times \sigma_{q}$, denoted by $\bar{\sigma}^{f}$, is as follows. The vertices of $\bar{\sigma}^{*}$ are the points $\bar{w}=\left(w_{j_{1}}, w_{j_{2}}, \cdots, w_{j_{q} q}\right)$ of $\bar{\sigma}^{\#}$. Write $\bar{w}<\bar{w}^{\prime}$ for any distinct $\bar{w}=\left(w_{j_{1} 1}, w_{j_{2} 2}, \cdots, w_{j_{q} q}\right), \bar{w}^{\prime}=\left(w_{j_{1}^{\prime}}, w_{j_{2}^{\prime} 2}, \cdots, w_{j_{q}^{\prime}}\right)$ in case that $w_{j_{k^{k}}} \leqq w_{j_{k^{k}}}, k=1,2, \cdots, q$. The $n$-simplexes of $\bar{\sigma}^{*}$ are those spanned by any $n+1$ points $\bar{w}_{0}<\bar{w}_{1}<\cdots<\bar{w}_{n}\left({ }^{4}\right)$. Let $K_{k}$ be ordered simplicial complexes, $k=1,2, \cdots, q$. It is then clear that the union of all $\bar{\sigma}^{\#}$ where $\sigma^{\#}=\sigma_{1} \times \sigma_{2} \times \cdots \times \sigma_{q}$ with $\sigma_{k}$ in $K_{k}$ constitutes a simplicial decomposition of

$$
K^{\#}=K_{1} \times K_{2} \times \cdots \times K_{q},
$$

which we shall denote by $\bar{K}^{\#}$.

It is easy to see that if $K$ is an ordered simplicial complex, $\Gamma$ acting on $K^{q}$ leaves the simplicial decomposition $\bar{K}^{q}$ invariant. Moreover, if $\gamma \in \Gamma$ leaves a simplex $\bar{\tau}$ in $\bar{K}^{q}$ spanned by $\bar{w}_{0}<\bar{w}_{1}<\cdots<\bar{w}_{n}$ invariant, then, since $\bar{w}_{i}<\bar{w}_{j}$ implies $\gamma\left(\bar{w}_{i}\right)<\gamma\left(\bar{w}_{j}\right), \gamma$ leaves every $\bar{w}_{i}$ invariant and hence leaves $\bar{\tau}$ pointwise invariant. It follows therefore that the identification map $\bar{I}: K^{q} \rightarrow K^{\Gamma}$ carries $\bar{K}^{q}$ naturally to a simplicial decomposition $\bar{K}^{\mathbf{T}}$ of $K^{\mathrm{r}}$.

In the remaining part of this section, we shall give some remarks on the homotopy type of $\Gamma$-products.

(1.1) If $f, f^{\prime}: X \rightarrow Y$ are homotopic maps between locally compact, paracompact Hausdorff spaces, then $f^{\mathrm{r}}$ and $f^{\prime \mathbf{r}}$ are homotopic.

In fact, let $F: X \times\langle 0,1\rangle \rightarrow Y$ be a homotopy connecting $f$ and $f^{\prime}$ (namely, a map such that $F(x, 0)=f(x), F(x, 1)=f^{\prime}(x)$ for any $\left.x \in X\right)$. Denote by $W$ the subset of $(X \times\langle 0,1\rangle)^{\mathbf{\Gamma}}$ of all points $\bar{I}\left(x_{1} \times t, x_{2} \times t, \cdots, x_{q} \times t\right), x_{i} \in X$, $t \in\langle 0,1\rangle$. We see then that $W$ is homeomorphic to $X^{\mathbf{r}} \times\langle 0,1\rangle$ in a natural manner and that the partial map $F^{\mathbf{\Gamma}} \mid W: W \rightarrow Y^{\mathbf{\Gamma}}$ is a homotopy connecting $f^{\Gamma}$ and $f^{\prime \Gamma}$.

(1.2) If $X$ and $Y$ have the same homotopy type, then $X^{\mathrm{r}}$ and $Y^{\mathrm{r}}$ have the same homotopy type.

In fact, let $f: X \rightarrow Y$ and $g: Y \rightarrow X$ be maps such that $g f \simeq_{l}$ (= the identity map) in $X$ and $f g \simeq_{\iota}$ in $Y$. Then, $g^{\Gamma} f^{\Gamma}=(g f)^{\Gamma} \simeq_{\iota}$ in $X^{\Gamma}$ and $f^{\Gamma} g^{\Gamma}=(f g)^{\Gamma} \simeq_{\iota}$ in $Y^{\mathbf{\Gamma}}$ by (1.1).

As an immediate consequence of (1.2), we have

(1.3) If $X$ is contractible, then $X^{\Gamma}$ is contractible.

We shall write $K_{m}$ for the $m$ th skeleton of any given simplicial complex $K$.

(1.4) The $\Gamma$-product of an m-connected complex is m-connected ( $m \geqq 0)$.

In fact, let $K$ be an oriented simplicial complex with a deformation of $K_{m}$ in $K$ into a point. Then, we verify easily that $\left(\bar{K}^{q}\right)_{m}$ is contained in $\left(K_{m}\right)^{q}$ and hence that $\left(\bar{K}^{q}\right)_{m}$ is deformable in $K^{q}$ into a point by a deformation in-

(4) This verification needs only some elementary treatment in analytical geometry. 
variant under $\Gamma$. It follows that $\bar{I}$ carries this deformation to a deformation in $K^{\Gamma}$ of the $m$ th skeleton of $\bar{K}^{\mathbf{r}}$ into a point. This proves (1.4).

2. Special cohomology. The cohomology theory over a locally compact, paracompact Hausdorff space $W$ is given as usual, based on a complete system of star-finite (open) coverings of $W$. (For convenience, we shall allow the repetition of the same open set as different members of the covering.) Let $\Sigma$ be a finite transformation group acting on $W$. We shall give some preliminaries on the cohomology arising from the fashion of $\Sigma$ acting on $W$ for later applications. Denote by $O(W, \Sigma)$ the orbit space over $W$ relative to $\Sigma$ and by $\bar{I}$ the identification map of $W$ to the orbit space. (If $\Sigma$ is a cyclic group with generator $T$, we shall write $O(W, \Sigma)$ as $O(W, T)$ in accordance with notation used in [3].) Denote also by $\Sigma^{\sharp}$ the integral group ring generated by the elements of $\Sigma$.

Let $\left\{V_{\lambda}\right\}$ be a complete system of star-finite coverings of $W$, invariant under $\Sigma$, such that, for each element $\gamma \in \Sigma$, and each member $A$ of $V_{\lambda}$, either $\gamma(A)$ and $A$ are disjoint or coincide. Let $K_{\lambda}$ be the nerve of $V_{\lambda}$. Let $W_{i}$ be a closed subset of $W$, invariant under $\Sigma$. We write $K_{i \lambda}$ for the subcomplex of $K_{\lambda}$ consisting of all simplexes whose nucleus meets $W_{i} . \Sigma$ acts then on $K_{\lambda}$ in a natural manner, leaving $K_{i \lambda}$ invariant, and, due to the construction of $\left\{V_{\lambda}\right\}$, we see that a projection $\pi=\pi_{\mu \nu}: K_{\mu} \rightarrow K_{\nu}$ invariant under $\Sigma$ can be chosen when $V_{\mu}$ refines $V_{\nu}$.

Let $\phi \in \Sigma^{*}$ and $\epsilon= \pm 1$. Let $W_{i} \supset W_{j}$ be closed subsets of $W$, invariant under $\Sigma$. $\phi$ gives then a cochain map $\phi$ in the group $C_{s}\left(K_{i \lambda}, K_{j \lambda} ; G\right)$ of $s$-cochains of the pair $\left(K_{i \lambda}, K_{j \lambda}\right)$ with coefficient group $G$. We shall write $\phi^{\epsilon} C_{s}\left(K_{i \lambda}, K_{j \lambda} ; G\right)$ as the image of this cochain map $\phi$ if $\epsilon=1$ and as its kernel if $\epsilon=-1$. More generally, if $\alpha$ is a set of $\phi^{\text {e's }}$ with $\phi \in \Sigma^{\#}, \epsilon= \pm 1$, we write ${ }^{\alpha} C_{s}\left(K_{i \lambda}, K_{j \lambda} ; G\right)$ as the intersection of the ${ }^{\phi \epsilon} C_{s}\left(K_{i \lambda}, K_{j \lambda} ; G\right)$ 's. The group ${ }^{\alpha} C_{s}\left(K_{i \lambda}, K_{j \lambda} ; G\right)$ for all $s$ (and a fixed $\lambda$ ) form a Mayer cochain complex $M_{\lambda}{ }^{(5)}$ and we may thus define the cohomology group of $M_{\lambda}$, to be denoted by ${ }^{\alpha} H^{s}\left(K_{i \lambda}, K_{j \lambda} ; G\right)$. We shall define the cohomology group ${ }^{\alpha} H^{s}\left(W_{i}, W_{j} ; G\right)$ as the direct limit in $\lambda$ of ${ }^{\alpha} H^{s}\left(K_{i \lambda}, K_{j \lambda} ; G\right)$, using the projection $\pi$ before. In a similar manner, we may define homology groups of this sort.

If $\alpha=\{\phi\}$ (i.e., $\alpha$ consists of one single element $\phi$ of $\left.\Sigma^{\sharp}\right)$, we write: ${ }^{\alpha} H^{s}\left(W_{i}, W_{j} ; G\right)$ as ${ }^{\phi} H^{s}\left(W_{i}, W_{j} ; G\right)$, and if $\alpha=\left\{\phi^{-1}\right\}$ we write ${ }^{\alpha} H^{s}\left(W_{i}, W_{j} ; G\right)$ as ${ }^{\phi^{-1}} H^{s}\left(W_{i} ; W_{j} ; G\right)$. Let $W_{1} \supset W_{2} \supset W_{3}$ be a triple of closed subsets of $W$, invariant under $\Sigma$. We verify easily the commutativity in each square of the following diagram (2.1), in which one of the row sequences is the cohomology sequence of the triple $W_{1} \supset W_{2} \supset W_{3}$, other two row sequences are defined in a similar manner as for the cohomology sequences of a triple, and all the homomorphisms in the column sequences are defined in the same way as in $[3$, p. 74$]$.

(5) See J. L. Kelley and E. Pitcher, Exact homomorphism sequences in homology theory, Ann. of Math. vol. 48 (1947) pp. 682-702. 


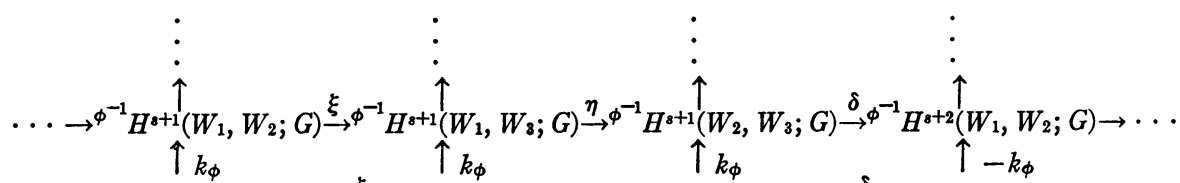

$$
\begin{aligned}
& \cdots \rightarrow \quad{ }^{\phi} H^{s}\left(W_{1}, W_{2} ; G\right) \stackrel{\xi}{\rightarrow}{ }^{\phi} H^{s}\left(W_{1}, W_{3} ; G\right) \stackrel{\eta}{\rightarrow}{ }^{\phi} H^{s}\left(W_{2}, W_{3} ; G\right) \stackrel{\delta}{\rightarrow}{ }^{\phi} H^{s+1}\left(W_{1}, W_{2} ; G\right) \rightarrow \cdots
\end{aligned}
$$

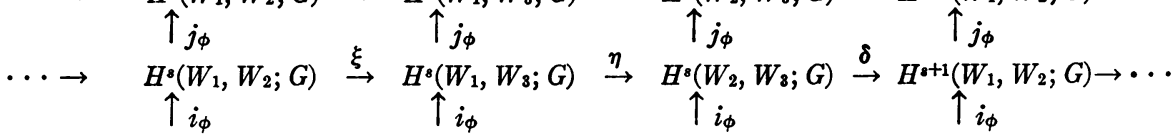

All these row and column sequences are exact.

Let $\phi_{1}, \phi_{2} \in \Sigma^{\sharp}$ be such that $\phi_{1} \phi_{2}=0$. Then, clearly, ${ }^{\phi_{2}} C_{s}\left(K_{i \lambda}, K_{j \lambda} ; G\right)$ $C^{\phi_{1}{ }^{-1}} C_{s}\left(K_{i \lambda}, K_{j \lambda} ; G\right)$ for each $\lambda$, and these inclusions give naturally a homomorphism

$$
\lambda:{ }^{\phi_{2}} H^{s}\left(W_{i}, W_{j} ; G\right) \rightarrow{ }^{\phi_{1}-1} H^{s}\left(W_{i}, W_{j} ; G\right) .
$$

We verify that, with $\xi, \eta, \delta, \lambda$ in (2.1) and (2.2),

$$
\lambda \xi=\xi \lambda, \quad \lambda \eta=\eta \lambda, \quad \lambda \delta=\delta \lambda .
$$

3. The isomorphism $\mu$, regular imbedding. We shall study a natural imbedding of $X$ into $X^{\mathbf{\Gamma}}$ and some relationship between cohomology groups of $X$ and of $X^{\mathbf{r}}$.

Consider again the space $W$ and the transformation group $\Sigma$ in $\$ 2$. We retain the usage of the notations there. When $\alpha$ consists of all $(1-a)^{-1}$ with $a \in \Sigma$, we write ${ }^{\alpha} H^{s}\left(W_{i}, W_{j} ; G\right)$ as ${ }^{\Sigma^{-1}} H^{s}\left(W_{i}, W_{j} ; G\right)$. We shall define a natural isomorphism

$$
\omega: H^{s}\left(O\left(W_{i}, \Sigma\right), O\left(W_{j}, \Sigma\right) ; G\right) \approx \Sigma^{-1} H^{s}\left(W_{i}, W_{j} ; G\right) .
$$

In fact, it is clear that the identification map $\bar{I}: W \rightarrow O(W, \Sigma)$ carries $\left\{V_{\lambda}\right\}$ into a complete system of coverings $\left\{\vec{I}\left(V_{\lambda}\right)\right\}$ of $O(W, \Sigma)$, and for each $\lambda, O\left(K_{\lambda}, \Sigma\right)$ is the nerve $\bar{I}\left(V_{\lambda}\right)$. Also, the identification map $K_{\lambda} \rightarrow O\left(K_{\lambda}, \Sigma\right)$ induces isomorphism: $H^{s}\left(O\left(K_{i \lambda}, \Sigma\right), O\left(K_{j \lambda}, \Sigma\right) ; G\right) \approx^{\Sigma^{-1}} H^{s}\left(K_{i}, K_{j} ; G\right)$. Taking direct limit in $\lambda$ by the projection $\pi$ gives the isomorphism (3.1).

Now, let $X$ be connected. We shall define for each $s$ an into-isomorphism

$$
\mu: H^{s}(X, G) \rightarrow H^{s}\left(X^{\Gamma}, G\right) .
$$

In fact, if $s=0$, both the groups in (3.2) are isomorphic to $G$ in a natural manner, and we define $\mu$ in a trivial way. We assume in the following that $s>0$. Let $\left\{V_{\lambda}\right\}$ be the system of coverings of $X^{q}$ invariant under the symmetric group $\Sigma_{q}$ of the $q$ letters $1,2, \cdots, q$, as given previously in $\$ 2$ (for $W$ and $\Sigma$ ). Let us write $\Sigma_{q-1}^{\prime}$ for the subgroup of $\Sigma_{q}$ of permutations which leaves 1 fixed, and write $e$ for the unit of the integral cohomology ring of an arbitrary locally compact, paracompact, connected Hausdorff space. If 
$v \in H^{s}(X, G)$, then the isomorphism $\omega$ in (3.1) for $\Sigma=\Sigma_{q-1}^{\prime}$ and $\left(W_{i}, W_{j}\right)$ $=\left(X^{q}, 0\right)$ carries $v \times e \in H^{s}\left(X \times X^{\Sigma_{q-1}}, G\right)$ into an element of ${ }^{\Sigma_{q-1}{ }^{\prime-1}} H^{s}\left(X^{q}, G\right)$. It follows that $v \times e \times \cdots \times e(q$ factors $) \in H^{s}\left(X^{q}, G\right)$ are represented by cocycles $z_{\lambda^{\prime}}$, determined by this isomorphism, over the nerves of some coverings $V_{\lambda^{\prime}}$ in $\left\{V_{\lambda}\right\}$, and invariant under $\Sigma_{q-1}^{\prime}$. Thus, if $T$ is a cyclic permutation of order $q$ in $\Sigma_{q}$, then $\sum_{i=0}^{q-1} T^{i}\left(z_{\lambda^{\prime}}\right)$ is invariant under $\Gamma$. Write $\theta(v)$ as the element of ${ }^{\Gamma^{-1}} H^{s}\left(X^{q}, G\right)$ represented by $\sum_{i=0}^{q-1} T^{i}\left(z_{\lambda^{\prime}}\right) . \theta(v)$ is then determined independently of the choice of $z_{\lambda^{\prime}}$, and $\theta: H^{s}(X, G) \rightarrow^{\Gamma^{-1}} H^{s}\left(X^{q}, G\right)$ is a homomorphism. Let us define

$$
\mu=\omega^{-1} \theta: H^{s}(X, G) \rightarrow H^{s}\left(X^{\Gamma}, G\right)
$$

where $\omega$ is the isomorphism (3.1) for $\Sigma=\Gamma$ and $\left(W_{i}, W_{j}\right)=\left(X^{q}, 0\right)$.

We assert that $\mu$ is an into-isomorphism. To see this, we associate $\theta^{\prime}(v)$ $=\sum_{i=0}^{q-1} T^{i}(v \times e \times \cdots \times e) \in H^{s}\left(X^{q}, G\right)$ to each $v \in H^{s}(X, G)$ with $T$ given above. Clearly, $\theta^{\prime}$ is an into-isomorphism: $H^{s}(X, G) \rightarrow H^{s}\left(X^{q}, G\right)$. By the definition of $\omega$, the commutativity of the diagram

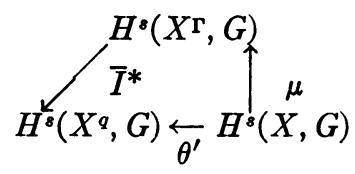

holds. Therefore, $\mu$ is an into-isomorphism.

We verify easily also the following statements (3.4) and (3.5) by the definition of $\mu$.

(3.4) Let $f: X \rightarrow Y$ be a map between locally compact, paracompact connected Hausdorff spaces. The commutativity of the diagram

$$
\begin{aligned}
& H^{s}(Y, G) \stackrel{\mu}{\rightarrow} H^{s}\left(Y^{\Gamma}, G\right) \\
& f^{*} \\
& H^{s}(X, G) \stackrel{\mu}{\rightarrow}\left(f^{\Gamma}\right)^{*} \\
& H^{s}\left(X^{\Gamma}, G\right)
\end{aligned}
$$

holds. The subgroup $\mu\left(H^{s}(X, G)\right)$ of $H^{s}\left(X^{\Gamma}, G\right)$ is invariant under homeomorphisms of $X$.

Let $\Gamma^{\prime}$ be a subgroup of $\Gamma$. Then, every orbit over $X^{q}$ relative to $\Gamma^{\prime}$ is contained in a certain orbit relative to $\Gamma$ and this gives a natural map $\eta_{\Gamma^{\prime} \Gamma}$ : $X^{\Gamma^{\prime}} \rightarrow X^{\Gamma}$.

(3.5) If $\Gamma^{\prime}$ is a subgroup of $\Gamma$, the commutativity of the diagram

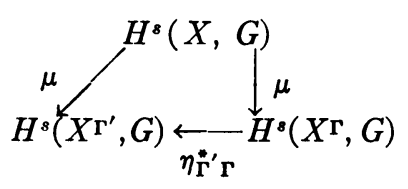

holds. 
The terminology "regularly imbedding of $X$ into $X^{\Gamma}$ " is used in the following sense. Let $s_{0}$ be an arbitrary point of $X$. We write

$$
s_{0}(X)=\bar{I}\left(X \times s_{0} \times \cdots \times s_{0}\right) \in X \boldsymbol{\Gamma} .
$$

Let $f_{s_{0}}: X \rightarrow X^{q}$ be the map which sends $x \in X$ to $\left(x, s_{0}, \cdots, s_{0}\right) \in X^{q}$. Let $f_{s_{0}}^{\prime}=\bar{I} f_{s_{0}}$. It is (regarded as) a homeomorphism of $X$ onto $s_{0}(X)$. Identifying $X$ and $s_{0}(X)$ under this homeomorphism, we say that $X$ is regularly imbedded in $X^{\Gamma}$ with reference point $s_{0}$. The commutativity of the diagram

$$
\underset{H^{s}\left(X^{q}, G\right) \underset{f_{s_{0}}^{*}}{H^{s}\left(X^{\mathrm{r}}, G\right)} \stackrel{\eta}{\rightarrow} H^{s}(X, G)}{\bar{I}^{*}\left(s_{0}(X), G\right)}
$$

holds, where the upper $\eta$ is the homomorphism induced by the inclusion $s_{0}(X) \subset X^{\Gamma}$. If $s>0$, for any $v \in H^{s}(X, G), f_{s_{0}}^{*}$ maps $\theta^{\prime}(v)$ to $v$ so that, by (3.3),

$$
\eta: \mu\left(H^{s}(X, G)\right) \approx H^{s}\left(s_{0}(X), G\right) .
$$

If $s=0$, this isomorphism is obviously true. Finally, let us state

(3.6) If $X$ is regularly imbedded in $X^{\Gamma}$, then $\eta \mu=$ the identity isomorphism of $H^{s}(X, G)$ for all $s$.

\section{THE REDUCED POWER OPERATION $P^{k}$ IN CYCLIC PRODUCTS OF SPHERES}

4. Remarks on periodic maps. Let $W$ be a finite-dimensional compact Hausdorff space and $T$ a periodic map of $W$ of prime period $p$ with fixed point set $F$. We shall give some remarks on the cohomology arising from the fashion of $T$ acting on $W$ for uses in later $\S \S 5,7$. It is assumed that the reader is somewhat familiar with the special homology theory on periodic maps $[3 ; 8]$. In general, we shall use a theory on $W$ module a closed subset of $W$ invariant under $T$. This generalization to the relative case can be easily obtained in a way parallel to that in [3]. For necessary definitions of relative special cohomology (and homology) groups, we may see $\$ 2$.

We shall write $Z$ and $Z_{p}$ respectively for the groups of integers and the group of integers $\bmod p$. The notations $\sigma, \tau, \rho, \bar{\rho}$, etc. will be referred to [3]. We denote by $W_{0}, W_{1}, W_{2}, W_{3}$ nonempty closed subsets of $W$ invariant under $T$ and by $E_{n}$ an $n$-cell with boundary sphere $S_{n-1}$.

(4.1) With $W, T, F, W_{0}$ given above, let the pair $\left(W, W_{0}\right)$ have the same cohomology groups mod $p$ as the pair $\left(E_{n}, S_{n-1}\right)$. Then:

(i) $H^{s}\left(F, W_{0} \cap F ; Z_{p}\right) \approx Z_{p}$ for $s=a$ certain $r \geqq 0$ and vanishes for other $s$.

(ii) $H^{s}\left(O(W, T), O\left(W_{0}, T\right) ; Z_{p}\right) \approx Z_{p}$ for $r+1<s \leqq n$ and vanishes for other $s$ in case $r<n ; H^{s}\left(O(W, T), O\left(W_{0}, T\right) ; Z_{p}\right) \approx Z_{p}$ for $s=r$ and vanishes for other $s$ in case $r=n$.

(4.1) is a generalization to the relative case of results in $[3, p .78]$ on 
periodic maps acting on a homology sphere. It can be proved in a way parallel to that for the absolute case. The detailed verification is thus omitted.

Let $\left(W, W_{0}\right)$ have the same integral cohomology groups as $\left(E_{n}, S_{n-1}\right)$. Then, $\left(W, W_{0}\right)$ has the same cohomology groups mod $p$ as $\left(E_{n}, S_{n-1}\right)$ by the usual relationship between cohomology groups given by the coefficient sequence $0 \rightarrow Z \rightarrow Z \rightarrow Z / p Z \rightarrow 0[3$, pp. 71-73]. It is easy to see that there is one integer $d(T)$ which is \pm 1 such that $T^{*}(v)=d(T) v$ for all $v \in H^{n}\left(W, W_{0} ; Z\right)$. If $d(T)=-1, p$ must be 2 .

(4.2) With $W, W_{0}, T$ given above such that $\left(W, W_{0}\right)$ has the same integral cohomology groups as $\left(E_{n}, S_{n-1}\right)$, and with $r$ given in (4.1), we have: $n-r$ is even or odd according as $d(T)=1$ or $=-1$. Moreover,

(i) If $d(T)=1, H^{s}\left(O(W, T), O\left(W_{0}, T\right) ; Z\right) \approx Z_{p}$ for $s=r+3, r+5, \cdots$, $n-1 ; \approx Z$ for $s=n$ and vanishes for other $s$.

(ii) If $d(T)=-1, H^{s}\left(O(W, T), O\left(W_{0}, T\right) ; Z\right) \approx Z_{p}$ for $s=r+3, r+5, \cdots, n$ and vanishes for other $s$.

This is the generalization to the relative case of results in $[3$, p. 82].

(4.3) With $W, W_{1}, W_{2}$ and $T$ given before, let $W_{1} \supset W_{2}$ and $i^{*}: H^{s}\left(W, W_{1} ; Z_{p}\right)$ $\approx H^{s}\left(W, W_{2} ; Z_{p}\right)$ for all $s$ where $i$ denotes the inclusion $\left(W, W_{2}\right) \subset\left(W, W_{1}\right)$. Then, $i^{*}: H^{s}\left(O(W, T), O\left(W_{1}, T\right) ; Z_{p}\right) \approx H^{s}\left(O(W, T), O\left(W_{2}, T\right) ; Z_{p}\right)$ for all $s$ where $\tilde{\imath}$ denotes the inclusion $\left(O(W, T), O\left(W_{2}, T\right)\right) \subset\left(O(W, T), O\left(W_{1}, T\right)\right)$.

By exactness property of cohomology sequence, it suffices to show that $H^{s}\left(O\left(W_{1}, T\right), O\left(W_{2}, T\right) ; Z_{p}\right)=0$ for all $s$, using that $H^{s}\left(W_{1}, W_{2} ; Z_{p}\right)=0$ for all $s$. Using arguments as in [3, pp. 74-77], it is easy to see that if $W$ has trivial cohomology groups mod $p$, then $O(W, T)$ has trivial cohomology groups $\bmod p$. Generalization of this to the relative case yields (4.3).

(4.4) With $W_{1}, W_{2}, W_{3}, T$ given before, suppose that (i) $W_{1} \supset W_{2} \supset W_{3}$, (ii) $\left(W_{i}, W_{i+1}\right)$ and $\left(E_{n_{i}}, S_{n_{i-1}}\right)$ have the same cohomology groups mod $p$ such that the integer $r_{i}$ given as in (4.1) corresponding to $\left(W_{i}, W_{i+1}\right)$ is less than $n_{i}-1, i=1,2$, and that $n_{2}<n_{1}$, and (iii) the coboundary homomorphism: $H^{n_{2}}\left(W_{2}, W_{3} ; Z_{p}\right) \rightarrow H^{n_{2}+1}\left(W_{1}, W_{2} ; Z_{p}\right)$ is trivial. Then, the coboundary homomorphism: $H^{s}\left(O\left(W_{2}, T\right), O\left(W_{3}, T\right) ; Z_{p}\right) \rightarrow H^{s+1}\left(O\left(W_{1}, T\right), O\left(W_{2}, T\right) ; Z_{p}\right)$ is trivial for all $s$.

In fact, using arguments as in [3, pp. 75-77], ${ }^{\circ} H^{s}\left(W_{i}, W_{i+1} ; Z_{p}\right) \approx Z_{p}$ for $r_{i}<s \leqq n$ and vanishes for other $s, i=1,2$, and

$$
\lambda:{ }^{\rho} H^{s}\left(W_{i}, W_{i+1} ; Z_{p}\right) \approx \bar{\rho}^{-1} H^{s}\left(W_{i}, W_{i+1} ; Z_{p}\right)
$$

$$
\text { for } r_{i}+1<s \leqq n_{i}, i=1,2 \text {, }
$$

where $\lambda$ is the homomorphism (2.2). We consider the diagram (2.1), and we have, in the row exact sequences in this diagram,

$$
\begin{aligned}
& j_{\rho}: H^{n_{i}}\left(W_{i}, W_{i+1} ; Z_{p}\right) \approx{ }^{\rho} H^{n_{i}}\left(W_{i}, W_{i+1} ; Z_{p}\right), \\
& k_{\rho}:{ }^{\rho} H^{s}\left(W_{i}, W_{i+1} ; Z_{p}\right) \approx{ }^{\rho^{-1}} H^{s+1}\left(W_{i}, W_{i+1} ; Z_{p}\right), \quad s \neq n_{i},
\end{aligned} \quad i=1,2 .
$$


By the hypothesis (iii), (4.6), and the commutativity property of the diagram (2.1), we get at once that $\delta:{ }^{\circ} H^{n_{2}}\left(W_{2}, W_{3} ; Z_{p}\right) \rightarrow{ }^{\circ} H^{n_{2}+1}\left(W_{1}, W_{2} ; Z_{p}\right)$ is a trivial homomorphism, so that, by (4.5) and (2.3), $\delta: \rho^{-1} H^{n_{2}}\left(W_{2}, W_{3} ; Z_{p}\right)$ $\rightarrow \rho^{-1} H^{n_{2}+1}\left(W_{1}, W_{2} ; Z_{p}\right)$ is a trivial homomorphism. Again, using this, (4.7), and the commutativity of $(2.1)$, we get that $\delta:{ }^{\circ} H^{n_{2}-1}\left(W_{2}, W_{3} ; Z_{p}\right)$ $\rightarrow{ }^{{ }^{n} \mathrm{n}_{2}}\left(W_{1}, W_{2} ; Z_{p}\right)$ is a trivial homomorphism. Continuing in this way with the aid of the hypothesis $n_{2}\left\langle n_{1}\right.$, we arrive at $\delta:{ }^{-1} H^{s}\left(W_{2}, W_{3} ; Z_{p}\right)$ $\rightarrow \rho^{-1} H^{s+1}\left(W_{1}, W_{2} ; Z_{p}\right)$ is trivial for all $s$. Taking $\rho=\tau$ and using the isomorphism (3.1) we complete the proof of (4.4).

\section{Cyclic products of spheres. We shall write}

$$
\vartheta_{n p}=\text { the } p \text {-fold cyclic product of an } n \text {-sphere } S_{n} \text {, }
$$

and denote by $T$ the periodic map used in the construction of $\vartheta_{n p}$. Only the case $p=$ prime will be considered in this paper. We shall calculate the integral cohomology groups of $\vartheta_{n p}(n \geqq 2)$ in this section. We denote by $J$ throughout the group $Z$ or the group $Z_{p}$.

We decompose $S_{n}$ into a cellular complex composed of a point $s_{0}$ and an open cell $C_{n}$ and consider the $p$-fold Cartesian product $\vartheta_{n p}^{0}$ of $S_{n}$ and the following subsets of $\vartheta_{n p}^{0}, \vartheta_{n p}$ :

$$
\begin{aligned}
& \Gamma_{n p}^{0}=\vartheta_{n p}^{0}-\Delta_{n p}^{0} \text { where } \Delta_{n p}^{0}=\text { the } p \text {-fold Cartesian product of } C_{n}, \\
& \Gamma_{n p}=\bar{I}\left(\Gamma_{n p}^{0}\right) .
\end{aligned}
$$

Since the fixed point set of $T$ is the diagonal sphere of $\vartheta_{n p}^{0}$, which meets $\Gamma_{n p}^{0}$ at one point, namely, the $p$-fold Cartesian product $s_{0}^{p}$ of $s_{0}, T\left(\Gamma_{n p}^{0}\right)=\Gamma_{n p}^{0}$ and $H^{s}\left(\vartheta_{n p}^{0}, \Gamma_{n p}^{0} ; J\right) \approx J$ for $s=n p$ and vanishes for other $s$, we have therefore by (4.1) and (4.2) that

$$
\begin{aligned}
& H^{*}\left(\vartheta_{n p}, \Gamma_{n p} ; Z\right) \approx \begin{cases}Z & s=n p \text { with } n p-n=\text { even, } \\
Z_{p} & \text { for } \quad \begin{array}{l}
s=n p \text { with } n p-n=\text { odd, } \\
Z_{p}
\end{array} \\
0 & s=n+2 k+1 \leqq n p \text { with } k>0, \\
\text { other } s,\end{cases} \\
& H^{s}\left(\vartheta_{n p}, \Gamma_{n p} ; Z_{p}\right) \approx\left\{\begin{array}{lll}
Z_{p} & \text { for } & s=0 \text { and } n+2 \leqq s \leqq n p, \\
0 & \text { other } s .
\end{array}\right.
\end{aligned}
$$

The given cellular decomposition of $S_{n}$ gives naturally a cellular decomposition of $\Gamma_{n p}^{0}$ composed of all $p$-fold products $B=B_{1} \times B_{2} \times \cdots \times B_{p}$ where each $B_{i}$ denotes either $s_{0}$ or $C_{n}$ and not all $B_{i}$ are $C_{n}$, and this yields a cellular decomposition of $\Gamma_{n p}$ with cells of the form $\bar{I}(B)$, since $T$ leaves only the points in the diagonal sphere of $\vartheta_{n p}^{0}$ fixed. In this cellular decomposition of $\Gamma_{n p}$, there is no $s$-cell if $s$ is not a multiple $n r$ of $n$ with $0 \leqq n r \leqq n(p-1)$ and there are $C_{p, r} / p s$-cells if $0<s=n r \leqq n(p-1)$ (where $C_{p, r}$ is the binomial coefficient). It is therefore easy to see that 


$$
H^{s}\left(\Gamma_{n p}, J\right) \approx \begin{cases}J & s=0, \\ \left\{J, C_{p, r} / p\right\} & \text { for } 0<s=n r \leqq n(p-1), n \geqq 2, \\ 0 & \text { other } s,\end{cases}
$$

where $\{J, t\}$ denotes the direct sum of $t$ groups each of which is isomorphic to $J$.

(5.4) The sequence

$$
0 \rightarrow H^{s}\left(\vartheta_{n p}, \Gamma_{n p} ; J\right) \stackrel{\xi}{\rightarrow} H^{s}\left(\vartheta_{n p}, J\right) \stackrel{\eta}{\rightarrow} H^{s}\left(\Gamma_{n p}, J\right) \rightarrow 0, \quad n \geqq 2,
$$

is exact $\left({ }^{6}\right)$, where $\xi, \eta$ are the injection and projection homomorphisms in the cohomology sequence of the pair $\left(\vartheta_{n p}, \Gamma_{n p}\right)$. Then,

$$
H^{*}\left(\vartheta_{n p}, J\right) \approx H^{*}\left(\vartheta_{n p}, \Gamma_{n p} ; J\right) \oplus H^{s}\left(\Gamma_{n p}, J\right), \quad n \geqq 2,
$$

with $H^{*}\left(\vartheta_{n p}, \Gamma_{n p} ; J\right), H^{s}\left(\Gamma_{n p}, J\right)$ given in (5.1)-(5.3).

We need prove that

$$
\eta: H^{s}\left(\vartheta_{n p}, J\right) \rightarrow H^{s}\left(\Gamma_{n p}, J\right)
$$

is onto for every $s$. This is obvious if $s$ is not a multiple $n r$ of $n$ with $0<n r$ $\leqq n(p-1)$. Let now $0<s=n r \leqq n(p-1)$. Since $T$ acting on $\Gamma_{n p}^{0}$ leaves only one point, namely $s_{0}^{p}$, fixed, using a simplicial decomposition of $\vartheta_{n_{p}}^{0}$ as described in $\$ 1$ with $s_{0}^{p}$ as a vertex, we verify straightforwardly that ${ }^{\sigma} H^{s}\left(\Gamma_{n p}^{0}, Z_{p}\right)$ $={ }^{r-1} H^{s}\left(\Gamma_{n p}^{0}, Z_{p}\right) \approx\left\{Z_{p}, C_{p, r} / p\right\}$ and that the homomorphism $j_{\sigma}: H^{s}\left(\Gamma_{n p}^{0}, Z_{p}\right)$ $\rightarrow^{\sigma} H^{s}\left(\Gamma_{n p}^{0}, Z_{p}\right)$ is onto with $j_{\sigma}$ given as in (2.1). Clearly, the projection homomorphism $\eta: H^{*}\left(\vartheta_{n p}^{0}, Z_{p}\right) \rightarrow H^{*}\left(\Gamma_{n p}^{0}, Z_{p}\right)$ is onto. Thus, by the commutativity property of (2.1), we have that $\eta:{ }^{\sigma} H^{s}\left(\vartheta_{n p}^{0}, Z_{p}\right) \rightarrow{ }^{\sigma} H^{s}\left(\Gamma_{n p}^{0}, Z_{p}\right)$ is onto with $\eta$ given as in (2.1). (5.6) for $J=Z_{p}$ follows now easily from (2.3) and the isomorphism (3.1). (5.6) for $J=Z$ follows from this and some usual relationship between cohomology groups given by the coefficient sequence $0 \rightarrow Z$ $\rightarrow Z \rightarrow Z / p Z \rightarrow 0\left({ }^{7}\right)$.

We shall write

$$
\begin{aligned}
Q^{s}\left(\vartheta_{n p}, J\right) & =\text { the kernel of } \eta \text { in }(5.5), \\
g_{n}^{*} & =\text { a generator of the infinite cyclic group } H^{n}\left(\vartheta_{n p}, Z\right), \\
g_{n}^{*} & =g_{n}^{*} \bmod p, \text { which generates } H^{n}\left(\vartheta_{n p}, Z_{p}\right) .
\end{aligned}
$$

(5.8) Let $n$ be even. Let $e_{n p}^{0}, e_{n p}$ be respectively generators of the infinite cyclic groups $H^{n p}\left(\vartheta_{n p}^{0}, Z\right), H^{n p}\left(\vartheta_{n p}, Z\right)$. We have $\bar{I}^{*}\left(e_{n p}\right)= \pm p e_{n p}^{0}$.

In fact, let us decompose $\vartheta_{n p}^{0}$ into a simplicial complex $K$ as in $\S 1$. The identification map $\bar{I}: \vartheta_{n p}^{0} \rightarrow \vartheta_{n p}$ carries $K$ into a simplicial decomposition of

(6) For $n=1$, this sequence is not exact in general. An example is $\vartheta_{12}$, which is the Möbius band.

( 7 ) Here, the assumption $n \geqq 2$ is needed. 
$\vartheta_{n p} . \vartheta_{n p}$ being a pseudo-manifold, $e_{n p}$ is represented by an oriented $n p$ simplex $\tau$, and there are exactly $p$ oriented $n p$-simplexes in $K$ which $\bar{I}$ maps into $\tau$. Since $n$ is even, it is easy to see that $T$ preserves orientation of the manifold $\vartheta_{n p}^{0}$. This gives (5.8).

6 . The cyclic reduced powers of $g_{n}^{\#}$. We assume $n \geqq 2$. Let $S_{n}$ be an oriented $n$-sphere. Let $e_{0}$ be the unit of the integral cohomology ring of $S_{n}$ and $e_{n}$ the generator of $H^{n}\left(S_{n}, Z\right)$ represented by $S_{n}$. Write $e_{n, i}^{0}=e_{0} \times \cdots$ $\times e_{0} \times e_{n} \times \cdots \times e_{0} \in H^{n}\left(\vartheta_{n p}^{0}, Z\right)$ where the $i$ th factor is $e_{n}$ and all other factors are $e_{0}$. Then, $e_{n p}^{0}= \pm \prod_{i=1}^{p} e_{n, s}^{0}$ with $e_{n p}^{0}$ given in (5.8) and a direct computation gives that, in $\vartheta_{n p}^{0},\left(\sum_{i=1}^{0} e_{n, i}^{0}\right)^{p}=0$ or $= \pm p ! e_{n p}^{0}$ according as $n$ is odd or even. Thus, by some properties of regular imbedding $S_{n}$ in $\vartheta_{n p}$ as given in $\S 3$, we see that $\bar{I}^{*}\left(g_{n}^{*}\right)= \pm \sum_{i=1}^{p} e_{n, \imath}^{0}$ so that, using (5.8),

$$
g_{n}^{* p}= \pm(p-1) ! e_{n p} \text { and hence } g_{n}^{\not p} \neq 0, \quad n=\text { even. }
$$

Following [9], $\Phi^{k}$ will be used to denote the cyclic reduced power operation $\bmod p$ (and for $p=2, P^{k}$ means the square operation $S q^{2 k}$ ). We have, in $\vartheta_{n p}$,

$$
\mathcal{P}^{k} g_{n}^{*} \neq 0, \quad 2 k \leqq n .
$$

Although this follows also from some general considerations in later sections, we may prove it, in a simple way, as follows. Let $n=2$. Then, $\mathcal{P}^{0} g_{2}^{*}=g_{2}^{\#} \neq 0$ and $\mathcal{P}^{1} g_{2}^{*}=g_{2}^{* p} \neq 0$ by (6.1). We shall complete the proof of (6.3) by induction on $n$. Let $E_{n+1}$ be an $(n+1)$-cell( $\left.{ }^{8}\right)$ with boundary $S_{n}$, and let $f: E_{n+1} \rightarrow S_{n+1}$ be the map which shrinks $S_{n}$ into a single point $s$ of $S_{n+1}$ and is topological elsewhere. Denote by $\vartheta_{n+1 p}^{\prime}$ the $p$-fold cyclic product of $E_{n+1}$. Then $\vartheta_{n p}$ $C \vartheta_{n+1 p}^{\prime}$, and $f$ gives rise naturally to a map $F: \vartheta_{n+1 p}^{\prime} \rightarrow \vartheta_{n+1 p}$ such that $F \bar{I}(x)$ $=\bar{I} f^{p}(x)$ where $x$ is an arbitrary point of the $p$-fold Cartesian product of $E_{n+1}$. Since $\vartheta_{n+1 p}^{\prime}$ is contractible by (1.3), the coboundary homomorphism

$$
\delta: H^{s}\left(\vartheta_{n p}, Z_{p}\right) \rightarrow H^{s+1}\left(\vartheta_{n+1 p}^{\prime}, \vartheta_{n p} ; Z_{p}\right), \quad s>0,
$$

is an into-isomorphism. Also, it is clear that $F\left(\vartheta_{n p}\right)=\bar{I}\left(s^{p}\right) \in \vartheta_{n+1 p}$ (with $s^{p}=$ the $p$-fold Cartesian product of $\left.s \in S_{n+1}\right)$. Thus, $F$ induces the homomorphism

$$
F^{*}: H^{s}\left(\vartheta_{n+1 p},\left(s^{p}\right) ; Z_{p}\right) \rightarrow H^{s}\left(\vartheta_{n+1 p}^{\prime}, \vartheta_{n p} ; Z_{p}\right) .
$$

We assert that $F^{*}$ is an onto-isomorphism for $s=n+1$. In fact, we have the into-homeomorphisms $f_{s}: S_{n+1} \rightarrow \vartheta_{n+1 p}\left(s \in S_{n+1}\right), f_{s_{0}}: E_{n+1} \rightarrow \vartheta_{n+1 p}^{\prime}\left(s_{0} \in S_{n}\right)$ as given in the last paragraph of $\S 3$. We verify easily that $F\left(f_{s_{0}}\left(S_{n}\right)\right)=s^{p}$ and $F$ maps $f_{s_{0}}\left(E_{n+1}-S_{n}\right)$ topologically onto $f_{s}\left(S_{n+1}-(s)\right)$. Then, by some property of regular imbedding given in $\$ 3$, the isomorphism (6.3) and some usual

(8) By a cell we mean a space which is homeomorphic to a bounded closed convex cell in a Euclidean space. 
arguments in cohomology theory, we prove our assertion.

Write $g_{n+1}^{\prime \prime}$ for the generator of $H^{n+1}\left(\vartheta_{n+1 p},\left(s^{p}\right) ; Z_{p}\right)$ such that $\xi_{g_{n+1}^{\prime *}}=g_{n+1}^{*}$ where $\xi$ is the injection homomorphism: $H^{s}\left(\vartheta_{n+1 p},\left(s^{p}\right) ; Z_{p}\right) \rightarrow H^{s}\left(\vartheta_{n+1}, Z_{p}\right)$. Then, if $2 k \leqq n, f^{p *} \mathcal{P}^{k} g_{n+1}^{\prime *}=\lambda \delta \mathcal{P}^{k} g_{n}^{\#}$ with $\lambda \in Z_{p}, \neq 0$ by our assertion above, and hence $P^{k} g_{n+1}^{\prime \prime} \neq 0$ by the isomorphism (6.3) and induction hypothesis. It follows that $\mathcal{P}^{k} g_{n+1}^{\#} \neq 0$. If $2 k=n+1$, then $\mathcal{P}^{k} g_{n+1}^{*}=g_{n+1}^{\sharp p} \neq 0$ by (6.1). This proves (6.2).

We assert also that

$$
\mathcal{P}^{k} g_{n}^{*} \in Q^{n+2 k(p-1)}\left(\vartheta_{n p}, Z_{p}\right), \quad k>0,
$$

with $Q^{n+2 k(p-1)}\left(\vartheta_{n p}, Z_{p}\right)$ given in (5.7), and by (5.4), it remains to show that $\eta \mathcal{P}^{k} g_{n}^{\#}=0$ for the case $n<n+2 k(p-1)=n r \leqq n(p-1)$. Write $d_{n r} \in H^{n r}\left(\Gamma_{n p}, Z_{p}\right)$ for the element represented by $\sum_{i=1}^{q} C_{n r}^{i}$ where $C_{n r}^{1}, C_{n r}^{2}, \cdots, C_{n r}^{q}$, $q=C_{p, r} / p$, are the oriented open $n r$-cells in $\Gamma_{n p}$ with respect to the cellular decomposition of $\Gamma_{n p}$ given in $\$ 5$ and with the orientation induced by the given orientation of $S_{n}$. In the $r$-fold Cartesian product $\vartheta_{n r}^{0}$ of $S_{n}$, let us denote by $e_{n, \imath}^{0}$ the element of $H^{n}\left(\vartheta_{n r}^{0}, Z_{p}\right)$ as given in the first paragraph of this section but with $Z$ replaced by $Z_{p}$ and with $p$ replaced by $r$. Since for each $j, j=1,2, \cdots, q$, we may construct a map $T_{j}: \vartheta_{n r}^{0} \rightarrow \Gamma_{n p}$ such that $T_{j}^{*}\left(C_{n r}^{j}\right)$ represents $\prod_{i=1}^{r} e_{n, i}^{0}$ and $T_{j}^{*}\left(S_{n}\right)$ represents $\sum_{i=1}^{r} e_{n, i}^{0}$, and since in $\vartheta_{n r}^{0}$ we have clearly $\mathcal{P}^{k} T_{j}^{*}\left(S_{n}\right)=0$, it follows therefore easily that $\eta \mathcal{P}^{k} g_{n}^{*}=0$. We get thus (6.4).

We shall write $S_{n}^{0}, S_{n}^{1}$ respectively for the diagonal spheres in $\vartheta_{n p}^{0}$ and $\bar{I}\left(S_{n}^{0}\right)$ in $\vartheta_{n p}$ and write

$$
\begin{array}{ll}
w_{n}^{*} \in H^{n}\left(S_{n}^{1}, Z\right) & \text { for the generator, } \\
w_{n}=w_{n}^{*} \quad(\bmod p) \in H^{n}\left(S_{n}^{1}, Z_{p}\right) . &
\end{array}
$$

By a consideration on the map $\bar{I}: \vartheta_{n p}^{0} \rightarrow \vartheta_{n p}$, we see easily that

$$
\zeta g_{n}^{*}= \pm p w_{n}^{*}, \quad \zeta g_{n}^{*}=0
$$

where $\zeta$ denotes the projection: $H^{n}\left(\vartheta_{n p}, J\right) \rightarrow H^{n}\left(S_{n}^{1}, J\right)$.

7. Neighbourhood of the diagonal sphere $S_{n}^{1}$ in $\vartheta_{n p}$. We intend to calculate the iterated cyclic powers of $g_{n}^{*}$. We recall the following: Let $\Delta_{(m, p)}$ be the lens space which is the orbit space over an $m$-sphere $S_{m}, m \geqq 2$, relative to an orthogonal transformation in $S_{m}$ of prime period $p$ without fixed point. The cohomology groups $H^{s}\left(\Delta_{(m, p)}, Z_{p}\right)$ of $\Delta_{(m, p)} \bmod p$ are isomorphic to $Z_{p}$ for $0 \leqq s \leqq m$. Let $v_{1} \in H^{1}\left(\Delta_{(m, p)}, Z_{p}\right), v_{2} \in H^{2}\left(\Delta_{(m, p)}, Z_{p}\right)$ be generators. Then, $v_{2}^{k}$ generates $H^{2 k}\left(\Delta_{(m, p)}, Z_{p}\right)$ for $0 \leqq 2 k \leqq m, v_{1} v_{2}^{k}$ generates $H^{2 k+1}\left(\Delta_{(m, p)}, Z_{p}\right)$ for $0 \leqq 2 k+1 \leqq m, v_{1}^{2}=0$, or $=v_{2}^{2}$ according as $p>2$ or $=2[17]$.

We may take $S_{n}$ as an analytical manifold. Then, naturally, $T$ acts on $\vartheta_{n p}^{0}$ and $S_{n}^{0}$ imbeds in $\vartheta_{n p}^{0}$ analytically. We may then consider the fiber bundle $\mathcal{B}_{0}$ 
over $S_{n}^{0}$ whose fibers are $(n p-n-1)$-dimensional normal spheres of $S_{n}^{0}$ in $\vartheta_{n p}^{0}$. Let $E_{0}, \phi_{0}, V_{0 x}$ be the total space, the projection, and the fiber over $x \in S_{n}^{0}$ of this bundle. Let $V_{0 x}^{\prime}, x \in S_{n}^{0}$, be the solid $(n p-n)$-sphere composed of the geodesics joining $x$ to points of $V_{0 x}$, and let $C_{0}=U_{x \in S_{n}}{ }^{\prime} V_{0 x}^{\prime}$, which is the map cylinder of $\phi_{0}$. The following are easily seen: $T$ leaves $E_{0}, C_{0}, V_{0 x}$, $V_{0 x}^{\prime}$ invariant, $T$ applied on $V_{0 x}$ is equivalent to an orthogonal transformation without fixed point. Thus, $\bar{I}$ carries $\mathcal{B}_{0}$ into a bundle $\mathbb{B}$ over $S_{n}^{\prime}$ with the lens space $\Delta_{(n p-n-1, p)}$ as fiber. Write $E=\bar{I}\left(E_{0}\right)$ and $C=\bar{I}\left(C_{0}\right) . C-E$ is an open subset of $\vartheta_{n p}$ containing $S_{n}^{1}$ and $S_{n}^{1}$ is a deformation retract of $C$.

We shall evaluate the cohomology groups $H^{s}\left(E, Z_{p}\right)$. To do this, we have to use arguments in the theory of periodic maps, although $B$ is a bundle so simple as over a sphere. Let $x_{0} \in S_{n}^{0}$ be a given point. For simplicity, write

$$
V_{0}=V_{0 x_{0}}, \quad V_{0}^{\prime}=V_{0 x_{0}}^{\prime}, \quad V=\bar{I}\left(V_{0}\right), \quad V^{\prime}=\bar{I}\left(V_{0}^{\prime}\right) .
$$

Using the theorem of Feldbau, we see easily that $C_{0}-E_{0}-V_{0}^{\prime}$ is an open $n p$-cell, and hence $H^{s}\left(C_{0}, E_{0} \cup V_{0}^{\prime} ; Z_{p}\right) \approx Z_{p}$ for $s=n p$ and vanishes for other $s$. Thus, since $T$ leaves $C_{0}$ and $E_{0} \cup V_{0}^{\prime}$ invariant, it follows from (4.1) that $H^{s}\left(C, E \cup V^{\prime} ; Z_{p}\right) \approx Z_{p}$ for $n+2 \leqq s \leqq n p$ and vanishes for other $s$. Applying similar arguments to the pair $\left(V_{0}^{\prime}, V_{0}\right)$, we find that $H^{s}\left(V^{\prime} \cup E, E ; Z_{p}\right)$ $\approx H^{s}\left(V^{\prime}, V ; Z_{p}\right) \approx Z_{p}$ for $2 \leqq s \leqq n p-n$ and vanishes for other $s$. Now, clearly, the coboundary homomorphism: $H^{s}\left(V_{0}^{\prime} \cup E_{0}, E_{0} ; Z_{p}\right) \rightarrow H^{s+1}\left(C_{0}, V_{0}^{\prime} \cup E_{0} ; Z_{p}\right)$ is trivial. Thus, using (4.4) we have the exact sequence

$$
0 \rightarrow H^{s}\left(C, V^{\prime} \cup E ; Z_{p}\right) \stackrel{\xi}{\rightarrow} H^{s}\left(C, E ; Z_{p}\right) \stackrel{\eta}{\rightarrow} H^{s}\left(V^{\prime} \cup E, E ; Z_{p}\right) \rightarrow 0,
$$

where $\xi$ and $\eta$ denote respectively the injection and projection homomorphism in the cohomology sequence of the triple $E \subset E \cup V^{\prime} \subset C$. Therefore,

$$
H^{s}\left(C, E ; Z_{p}\right) \approx \begin{cases}Z_{p} & 2 \leqq s \leqq n+1, n p-n+1 \leqq s \leqq n p, \\ Z_{p} \oplus Z_{p} & \text { for } n+2 \leqq s \leqq n p-n, \\ 0 & \text { other } s .\end{cases}
$$

We shall write

$$
Q^{\circ}\left(C, E ; Z_{p}\right)=\text { the kernel of } \eta \text { in (7.1). }
$$

Let

$$
\bar{w}_{n}=\phi^{*}\left(w_{n}\right) \in H^{n}\left(E, Z_{p}\right)
$$

where $w_{n}$ is given in (6.5) and $\phi$ denotes the projection of the bundle $\mathcal{B}$. We assert that $\bar{w}_{n} \neq 0$. In fact, if $p>2$, then the cross section of $\mathcal{B}_{0}$ and hence the cross section of $\mathbb{B}$ exists since $n p-n-1 \geqq n$; our assertion is thus obvious. Assume $p=2$. We observe that the intersection number $\bmod 2$ in $\vartheta_{n 2}^{0}$ of the fundamental $n$-cycle of $S_{n}^{0}$ with itself is 0 . If $a_{n} \in H^{n}\left(\vartheta_{n 2}^{0}, Z_{2}\right)$ is dual to this fundamental cycle, then $a_{n}^{2}=0$; it follows that the fundamental character- 
istic class mod 2 of $\mathcal{B}$ vanishes [13]. This proves that $\phi_{0}^{*}: H^{s}\left(S_{n}^{0}, Z_{2}\right)$ $\rightarrow H^{s}\left(E_{0}, Z_{2}\right)$ is an into-isomorphism. Our assertion follows easily. Now, since $S_{n}^{1}$ is a deformation retract of $C$, by (7.2) and the exactness of the cohomology sequence of the pair $(C, E)$, we have that

$$
H^{s}\left(E, Z_{p}\right) \approx\left\{\begin{array}{lll}
Z_{p} & 0 \leqq s \leqq n-1, n p-n \leqq s \leqq n p-1, \\
Z_{p} \oplus Z_{p} & \text { for } & n \leqq s \leqq n p-n-1, \\
0 & \text { other } s .
\end{array}\right.
$$

Using (7.5), the cohomology ring mod $p$ of a lens space, and Wang sequence of the bundle $B[6$, p. 471$]$, namely,

$$
\begin{aligned}
\ldots & \stackrel{\alpha}{\rightarrow} H^{s}\left(E, Z_{p}\right) \stackrel{\beta}{\rightarrow} H^{s}\left(\Delta_{(n p-n-1, p)}, Z_{p}\right) \\
& \stackrel{d_{n}}{\rightarrow} H^{n}\left(S_{n}^{1}, Z_{p}\right) \otimes H^{s-n+1}\left(\Delta_{(n p-n-1, p)}, Z_{p}\right) \\
& \stackrel{\alpha}{\rightarrow} H^{s+1}\left(E, Z_{p}\right) \stackrel{\beta}{\rightarrow} \cdots,
\end{aligned}
$$

we can find easily the cohomology ring of $E \bmod p$. Notice that the homomorphism $\beta$ in (7.6) may be interpreted geometrically as the projection of the cohomology groups of $E$ into those of an arbitrary fiber, and satisfies the relation $\beta(x y)=\beta(x) \beta(y)$. One observes that there are

$$
\bar{v}_{1} \in H^{1}\left(E, Z_{p}\right), \bar{v}_{2} \in H^{2}\left(E, Z_{p}\right) \text { such that } \beta\left(\bar{v}_{1}\right)=v_{1}, \beta\left(\bar{v}_{2}\right)=v_{2} \text {, }
$$

and hence that the homomorphism $d_{n}$ in (7.6) is trivial since $v_{1}$ and $v_{2}$ generates the cohomology ring of $\Delta_{(n p-n-1, p)} \bmod p$. Thus, some usual arguments in spectral homology theory and, in particular, those to establish the sequence (7.6) together with (7.7) prove that $E$ has the same cohomology ring $\bmod p$ of the product $S_{n}^{1} \times \Delta_{(n p-n-1, p)}$.

We shall write also

$$
Q^{s}\left(E, Z_{p}\right)=\text { the kernel of the homomorphism } \beta \text { in (7.6). }
$$

The pair $(C, E)$ has cohomology ring $\bmod p$ with trivial multiplication (i.e., we have always $x y=0$ for any cohomology classes $x, y \bmod p$ of the pair $(C, E)$ ). In fact, since $C$, up to a homeomorphism, is the space $S_{n}^{1} \cup E$ $\times\langle 0,1\rangle$ with the topology obtained by identifying, in $E \times\langle 0,1\rangle$, the subset $E \times(0)$ to $S_{n}^{1}$ in a natural manner by means of the projection $\phi: E \rightarrow S_{n}^{1}$ of $\mathcal{B}$, we notice that the multiplication of the cohomology ring of the pair $(E \times\langle 0,1\rangle$, $E \times((0) \cup(1))$ is trivial.

8. A basic relation. We shall write

$$
M=\vartheta_{n p}-(C-E)
$$

and consider the following diagram (4.1) in which $\iota_{0}, \iota_{1}$ etc. are homomorphisms induced by inclusion $E \subset M,(M, E) \subset\left(\vartheta_{n p}, C\right)$, etc., and $\delta, \delta^{\prime}$ 
are coboundary homomorphisms.

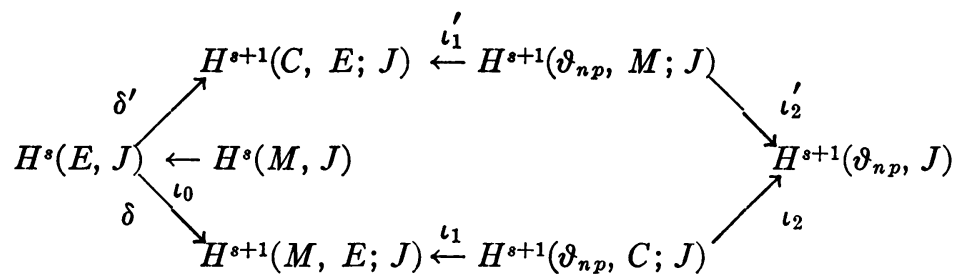

Clearly, $\iota_{1}$ and $\iota_{1}^{\prime}$ are onto-isomorphisms by excision, and the commutativity

$$
\iota_{2} \iota_{1}^{-1} \delta=\iota_{2}^{\prime} \iota_{1}^{\prime-1} \delta^{\prime}
$$

holds by some usual arguments. Also, using the exactness of the cohomology sequences of the pair $(C, E)$ and $\left(\vartheta_{n p}, M\right)$, the assumption $n \geqq 3$, and (5.4), (7.2), we see that $H^{1}\left(M, Z_{p}\right), H^{2}\left(M, Z_{p}\right)$ are cyclic groups of order $p$ with generators

$$
b_{1} \in H^{1}\left(M, Z_{p}\right), \quad b_{2} \in H^{2}\left(M, Z_{p}\right)
$$

such that

$$
\iota_{0}\left(b_{1}\right)=\bar{v}_{1}, \quad \iota_{0}\left(b_{2}\right)=\bar{v}_{2} .
$$

Finally, since $S_{n}^{1}$ is a deformation retract of $C$, we verify by (6.6) that

$$
\begin{aligned}
\iota_{2} \iota_{1}^{-1}: H^{8}\left(M, E ; Z_{p}\right) & \approx H^{8}\left(\vartheta_{n p}, Z_{p}\right) \quad \text { for all } s \neq 0, n+1, \\
H^{n+1}\left(M, E ; Z_{p}\right) & \approx Z_{p} .
\end{aligned}
$$

We shall write

$$
\begin{gathered}
a_{n+1}=\mu \delta \bar{w} \bar{w}_{n} \in \bar{w} H^{n+1}\left(M, E ; Z_{p}\right) \\
g_{n} \in H^{n}\left(M, E ; Z_{p}\right)
\end{gathered}
$$

with $\mu \in Z_{p}, \neq 0$, with $\iota_{2} \iota_{1}^{-1}\left(g_{n}\right)=\stackrel{\sharp}{g_{n}}$.

We assert that

$$
Q^{s+1}\left(\vartheta_{n p}, Z_{p}\right)=\iota_{2} \iota_{1}^{-1} \delta Q^{8}\left(E, Z_{p}\right)
$$

where $Q^{s+1}\left(\vartheta_{n p}, Z_{p}\right)$ and $Q^{s}\left(E, Z_{p}\right)$ are given in (5.7), (7.8). In fact, it is easy to verify first that $Q^{s+1}\left(C, E ; Z_{p}\right)=\delta^{\prime} Q^{s}\left(E, Z_{p}\right)$ with $Q^{s}\left(C, E ; Z_{p}\right)$ given in (7.3). In $\vartheta_{n p}^{0}$, we may take an open $n p$-cell $W \subset \Delta_{n p}^{0} \cap\left(C_{0}-E_{0}-V_{0}\right)$ which $T$ leaves invariant. Then, since the inclusions $\left(C_{0}, C_{0}-W\right) \subset\left(C_{0}, E_{0} \cup V_{0}\right)$ and $\left(\vartheta_{n p}^{0}, \vartheta_{n p}^{0}-W\right) \subset\left(\vartheta_{n p}^{0}, \Gamma_{n p}^{0}\right)$ induce obviously onto-isomorphisms: $H^{*}\left(C_{0}, E_{0}\right.$ $\left.\cup V_{0} ; Z_{p}\right) \approx H^{s}\left(C_{0}, C_{0}-W ; Z_{p}\right)$ and $H^{s}\left(\vartheta_{n p}^{0}, \Gamma_{n p}^{0} ; Z_{p}\right) \approx H^{s}\left(\vartheta_{n p}^{0}, \vartheta_{n p}^{0}-W ; Z_{p}\right)$, it follows from (4.3) that the inclusions $(C, C-\bar{I}(W)) \subset(C, E \cup V)$ and $\left(\vartheta_{n p}, \vartheta_{n p}-\bar{I}(W)\right) \subset\left(\vartheta_{n p}, \Gamma_{n p}\right)$ induce onto-isomorphism: $H^{s}\left(C, E \cup V ; Z_{p}\right)$ $\approx H^{s}\left(C, C-\bar{I}(W) ; Z_{p}\right)$ and $H^{s}\left(\vartheta_{n p}, \Gamma_{n p} ; Z_{p}\right) \approx H^{s}\left(\vartheta_{n p}, \vartheta_{n p}-\bar{I}(W) ; Z_{p}\right)$. This gives $\iota_{2}^{\prime} \iota_{1}^{\prime-1}: Q^{s}\left(C, E ; Z_{p}\right) \approx Q^{s}\left(\vartheta_{n p}, Z_{p}\right)$ by definition of these groups and some 
usual arguments. Then, using (8.2) we complete the proof of (8.6).

We shall write

$$
\begin{array}{ll}
a_{n+2 k+1}^{*}=\iota_{2} \iota_{1}^{-1}\left(a_{n+1} b_{2}^{k}\right) \in H^{n+2 k+1}\left(\vartheta_{n p}, Z_{p}\right) & \text { for } 0 \leqq 2 k \leqq n p-n-1, \\
a_{n+2 k+2}^{\sharp}=\iota_{2} \iota_{1}^{-1}\left(a_{n+1} b_{1} b_{2}^{k}\right) \in H^{n+2 k+2}\left(\vartheta_{n p}, Z_{p}\right) & \text { for } 0 \leqq 2 k \leqq n p-n-2 .
\end{array}
$$

Clearly, $\bar{v}_{n}=\phi^{*}\left(w_{n}\right) \in Q^{s}\left(E, Z_{p}\right)$, so $\bar{w}_{n} \bar{v}_{2}^{k} \in Q^{n+2 k}\left(E, Z_{p}\right), \bar{w}_{n} \bar{v}_{1} \bar{v}_{2}^{k} \in Q^{n+2 k+1}\left(E, Z_{p}\right)$. Then, since $\delta\left(\bar{w}_{n} \bar{v}_{2}^{k}\right)=\mu a_{n+1} b_{2}^{k}, \delta\left(\bar{w}_{n} \bar{v}_{1} \bar{v}_{2}^{k}\right)=\mu a_{n+1} b_{1} b_{2}^{k}$, by (8.6) the $a_{s}^{*}$ in (8.7) generates the cyclic group $Q^{s}\left(\vartheta_{n p}, Z_{p}\right)$ and the $a_{n+1} b_{2}^{k}, a_{n+1} b_{1} b_{2}^{k}$ in (8.7) are not zero.

We shall establish the basic relation:

$$
g_{n} b_{2}=a_{n+1} b_{1}
$$

with $a_{n+1}$ in (8.5) suitably chosen. In fact, since $b_{1}, b_{2}$ generates respectively $H^{1}\left(M, Z_{p}\right), H^{2}\left(M, Z_{p}\right)$, using some usual arguments in cohomology theory, we conclude that there is

$$
b_{2}^{*} \in H^{2}(M, Z)
$$

of order $p$ such that $b_{2}=b_{2}^{*} \bmod p$ and $\delta^{*} b_{1}=\lambda b_{2}^{*}$ with $\lambda \in Z, \neq 0$, where $\delta^{*}$ is the coboundary homomorphism of the coefficient sequence $0 \rightarrow Z \rightarrow Z$ $\rightarrow Z / p Z \rightarrow 0$. Also, since $S_{n}^{1}$ is a deformation retract of $\vartheta_{n p}$, by (5.4) and (6.6) we have $H^{n+2}(M, E ; Z)=H^{n}(M, E ; Z)=0$ and $H^{n+1}(M, E ; Z) \approx Z_{p}$. Let $a_{n+1}^{*}$ $\in H^{n+1}(M, E ; Z)$ be a generator and let $b_{n+3}^{*}=a_{n+1}^{*} b_{2}^{*}$. Then $b_{n+3}^{*} \neq 0$ and $p b_{n+3}^{*}=0$. For, $a_{n+1}^{*} b_{2}^{*} \bmod p$ is $\nu a_{n+1} b_{2}$ with $\nu \in Z_{p}, \neq 0$, and $a_{n+1} b_{2} \neq 0$. That $p b_{n+3}^{*}=0$ is obvious.

Let us choose a covering of $M$ with nerve $N$ such that $b_{2}^{*}, a_{n+1}^{*}$ are represented by cocycles $x_{2} \in C_{2}(N, Z), x_{n+1} \in C_{n+1}\left(N, N_{0} ; Z\right)$ with $p x_{2} \sim 0, p x_{n+1} \sim 0$ where $N_{0}$ denotes the subcomplex of $N$ consisting of all simplexes whose nucleus meets $E$. Let $x_{1} \in C_{1}(N, Z), x_{n} \in C_{n}\left(N, N_{0} ; Z\right)$ with $\delta x_{1}=p x_{2}, \delta x_{n}$ $=p x_{n+1}$. Then, $x_{1}, x_{2}, x_{n}, x_{n+1}$ mod $p$ represent respectively $\lambda_{1} b_{1}, \lambda_{2} b_{2}, \lambda_{3} g_{n}$, $\lambda_{4} a_{n+1}$ with $\lambda_{i} \in Z_{p}, \neq 0$, and $x_{n+1} x_{2}$ represents $\omega b_{n+3}^{*}$ with $\omega \in Z, \neq 0$. Now $H^{n+2}\left(M, E ; Z_{p}\right) \approx Z_{p}$ is generated by $a_{n+2}$; clearly $\delta^{*} a_{n+2}=\lambda^{\prime} b_{n+3}^{*}$. Thus, since $\delta\left(x_{n+1} x_{1}\right)=(-)^{n} p x_{n+1} x_{2}$, and $\delta\left(x_{n} x_{2}\right)=p x_{n+1} x_{2}, g_{n} b_{2}=\sigma a_{n+1} b_{1}, \sigma \in Z_{p}, \neq 0$. Write $\sigma a_{n+1}$ simply as $a_{n+1}$ so that we get (8.8).

Finally, let us remark here that $a_{n+1}$ in (8.5) will be so chosen that (8.8) holds. It is obvious from (8.8) that

$$
g_{n} b_{2}^{k+1}=a_{n+1} b_{1} b_{2}^{k}
$$

9. Cyclic reduced powers of $Q^{s}\left(\vartheta_{n p}, Z_{p}\right)$. This section contains only computations. Explicit relations between iterated powers are given for $Q^{s}\left(\vartheta_{n p}, Z_{p}\right)$.

(I) $p=2$. It is easy to see from (5.4) that the Bockstein homomorphism maps $H^{2 k}\left(S_{n} * S_{n}, Z_{2}\right)$ onto $H^{2 k+1}\left(S_{n} * S_{n}, Z_{2}\right), 2 \leqq 2 k<n$. Thus, by (5.4) and (6.2), 


$$
S q^{1} g_{n}^{\#}=0, \quad S q^{i} g_{n}^{*}=\stackrel{\star}{*} a_{n+j}
$$$$
2 \leqq j \leqq n .
$$

We verify $b_{1}^{2}=b_{2}$, using (7.7), (8.3). As in [11], we have $S q^{i} b_{1}^{Q}=c\left(j, q ; Z_{2}\right) b_{1}^{a+j}$ where $c\left(j, q ; Z_{2}\right)$ is the mod 2 value of the hinomial coefficient $C_{q, j}$ (with the convention: $C_{q, j}=0$ if $\left.j>q\right)$. Write $a_{n+1+q}=a_{n+1} b_{1}^{q}$. Then, since $S q^{j} a_{n+1}$ $=\delta S q^{i} \bar{w}_{n}=\delta \phi^{*} S q^{i} w_{n}=0$ for $j>0$, the formula of Cartan gives $S q^{i} a_{n+1+q}$ $=c\left(j, q ; Z_{2}\right) a_{n+1+q+j}, q+j+1 \leqq n$. Thus, using (8.7),

$$
S q^{j} a_{n+1+q}^{\star}=c\left(j, q ; Z_{2}\right) \stackrel{a_{n+1+q+j}}{*}, \quad q+j+1 \leqq n .
$$

We have $\left({ }^{9}\right)$

$$
S q^{i} g_{n}^{\#}=\cdot q^{i^{i_{1}}} S q^{2^{i_{2}}} \cdots S q^{2^{i_{j}}{ }^{\prime \prime}}{ }_{n}^{\#}
$$$$
2 \leqq j \leqq n,
$$

for some integers $i_{j^{\prime}}>i_{j^{\prime}-1}>\cdots>i_{1} \geqq 0$. In fact, if $j$ is a power of 2 , there is nothing to prove. If this is not the case, we may write $j=2^{k}(2 r+1)$ with $r$ $>0$, and hence, using (9.1), (9.2), and the argument in [11], $S q^{i} g_{n}^{*}=a_{n+j}^{*}$ $=S q^{2^{k}} a_{n+j-2^{k}}^{f}=S q^{2^{k}} S q^{j-2^{k}} g_{n}^{f}$. An induction on $j$ completes the proof.

It is easily verified by (9.1), (9.2) that the following (9.4) always holds:

$$
S q^{i} S q^{2 k-j} g_{n}^{\dagger}=0
$$$$
\text { for } 0<j<2^{k} \text {. }
$$

(II) $p>2$. If $n=3, \mathscr{P}^{k} g_{n} \neq 0$ only when $k=0,1$ and $\mathcal{P}^{1} g_{n}^{*}=\lambda a_{n+2 p-2}^{*}$ with $\lambda \in Z_{p}, \neq 0$ by (6.2), (6.4). Hereafter, we assume $n \geqq 4$.

We verify first that $P^{k} a_{n+1} \neq 0$ and $P^{k} b_{1} \neq 0$ only when $k=0$ so that $\Phi^{k} a_{n+1} b_{1}$ $\neq 0$ only when $k=0$ by the formula of Steenrod-Cartan [9]. We have $\mathcal{P}^{k} b_{2}^{q}$ $=c\left(k, q ; Z_{p}\right) b_{2}^{q+k(p-1)}$ where $2(q+k(p-1)) \leqq n p-n-1$ and $c\left(k, q ; Z_{p}\right)$ is the mod $p$ value of the binomial coefficient $C_{q, k}$. Also, $\Phi^{k} b_{2} \neq 0$ only when $k=0,1$ and $P^{1} b_{2}=b_{2}^{p}$.

Using the relation (8.8) and the formula of Steenrod-Cartan,

$$
\left(\mathcal{P}^{k} g_{n}\right) b_{2}+\left(\Phi^{k-1} g_{n}\right) b_{2}^{p}=0,
$$

$k>0$.

Then, since $\mathcal{P}^{k} g_{n}^{*} \in Q^{n+2 k(p-1)}\left(\vartheta_{n p}, \quad Z_{p}\right)$, by (8.4) we conclude $\mathcal{P}^{k} g_{n}$ $=-\Phi^{k-1} g_{n} b_{2}^{p-1}, k>0$. It follows therefore from (8.9), (8.7), and (9.5) that

$$
\mathcal{P}^{k} g_{n}^{*}=(-)^{k} a_{n+2 k(p-1)}^{*},
$$

$$
2 \leqq 2 k \leqq n .
$$

With arguments parallel to that used in the case (I) before, we deduce that

$$
\begin{aligned}
& \mathcal{P}^{k} a_{n+2 q+1}^{\sharp}=c\left(k, q ; Z_{p}\right) a_{n+2(q+k(p-1))+1}^{\#}, 2(q+k(p-1)) \leqq n(p-1)-1 . \\
& \mathcal{P}^{k} a_{n+2 q+2}^{+}=c\left(k, q ; Z_{p}\right) a_{n+2(q+k(p-1))+2}^{*}, 2(q+k(p-1)) \leqq n(p-1)-2 .
\end{aligned}
$$

A criterion for evaluating $c\left(k, q ; Z_{p}\right)$ for $p=2$ was given in [11]. A straight-

( ${ }^{9}$ See Adem [1]. Generalizations of results in [1] to cyclic reduced powers $P^{k} \bmod p$ with $p$ prime $>2$ had been also obtained by Adem. 
forward generalization to any prime $p$ is the following: Let

$$
k=\sum_{i=0}^{m} a_{i} p^{i}, \quad q=\sum_{i=0}^{m} b_{i} p^{i}
$$

be the $p$-adic expansion of $k, q$. Then $c\left(k, q ; Z_{p}\right)=c\left(a_{1}, b_{1} ; Z_{p}\right) c\left(a_{2}, b_{2} ; Z_{p}\right) \cdots$ $c\left(a_{m}, b_{m} ; Z_{p}\right)$. Thus,

(9.9) $c\left(k, q ; Z_{p}\right) \neq 0$ if and only if, in the p-adic expansions (5.8) of $k, q$, $a_{i} \leqq b_{i}, i=0,1, \cdots, m$.

(9.10) $\mathcal{P}^{k} g_{n}^{\#}, 2 \leqq 2 k \leqq n$, can be expressed as

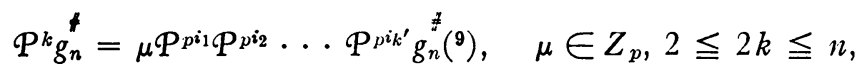

for some integers $i_{k^{\prime}} \geqq i_{k^{\prime}-1} \geqq \ldots \geqq i_{1} \geqq 0$ where the number of $i_{j}$ 's which are equal to a given $i^{\prime}$ is just the coefficients $a_{i^{\prime}}$ in the p-adic expansion (9.8) of $k$.

In fact, if $k$ is a power of $p$, there is nothing to prove. Suppose that this is not the case. Let $i_{1}$ be the least $i$ such that $a_{i} \neq 0$ with $k$ written as in (5.8), and let $r=\sum_{i=i_{0}+1}^{m} a_{i} p^{i-\left(i_{1}+1\right)}$. Then, either (i) $a_{i_{1}}>1$ or (ii), $a_{i_{1}}=1$, $r \geqq 1$. Write $t=k(p-1)-1$. In case (i), a simple calculation gives $t-p^{i_{1}}(p-1)$ $=p^{i_{1}+1}\left(r+a_{i_{1}}-2\right)+\left(p-a_{i_{1}}\right) p^{i_{1}}+\sum_{i=0}^{i_{1}-1}(p-1) p^{i}$ with $r+a_{i_{1}}-2 \geqq 0, p>p$ $-a_{i_{1}}>0$. It follows therefore from (9.9) that $c\left(p^{i_{1}}, t-p^{i_{0}}(p-1) ; Z_{p}\right) \neq 0$, and hence from (9.5), (9.6) that

$$
\mathcal{P}^{k} g_{n}^{\star}=\mu^{\prime} \Psi^{p_{1}} \Phi^{k-p^{i_{1}}} g_{n}^{*},
$$

In case (ii), a simple calculation gives $t-p^{i_{1}}(p-1)=p^{i_{0}+1}(r(p-1)-1)$ $+\sum_{i=0}^{i_{0}}(p-1) p^{i}$, and hence, using similar arguments as before, we have (9.12). An induction on $k$ completes the proof of our statement.

(9.13) The expression

$$
\mathcal{P}^{k} g_{n}^{\#}=\mu \mathcal{P}^{p_{1} 1} \Phi^{p_{2}} \ldots \mathcal{P}^{p^{i k^{\prime}}} g_{n}^{\#}, \quad \mu \in Z_{p}, 2 \leqq 2 k \leqq n,
$$

for some $i_{k^{\prime}} \geqq i_{k^{\prime}-1} \geqq \cdots \geqq i_{1} \geqq 0$ is unique.

We need prove that any expression

$$
\mathcal{P}^{k} g_{n}{ }^{\#}=\mu^{\prime} \mathcal{P}^{p^{\prime \prime} 1} \mathcal{P}^{p^{i}{ }_{2}} \ldots \mathcal{P}^{p^{\prime \prime} k^{\prime \prime}} g_{n}^{\#}, \quad \mu^{\prime} \in Z_{p}, 2 \leqq 2 k \leqq n,
$$

for some $i_{\boldsymbol{k}^{\prime \prime}}^{\prime} \geqq i_{k^{\prime \prime}-1}^{\prime} \geqq \ldots . \geqq i_{1}^{\prime} \geqq 0$ is the same as that in (9.10). It is easy to see that, in (9.11), (9.14), $k=\sum_{j=1}^{k^{\prime}} p^{i j}=\sum_{j=1}^{k^{\prime \prime}} p^{i^{\prime} i}$, and we assert $i_{1}^{\prime}=i_{1}$. In fact, if this is not the case, e.g., $i_{1}^{\prime}<i_{1}$, we shall have $i_{1}^{\prime}=i_{2}^{\prime}=\cdots=i_{p}^{\prime}$, and a simple calculation, using (9.5), (9.6) will give that, in (9.14), $\mathcal{P}^{k} g_{n}^{*}=0$, contradicting a well-established result. Therefore $i_{1}^{\prime}=i_{1}$, and hence

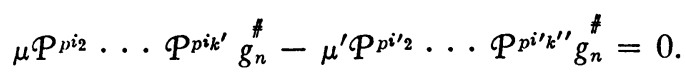

Thus, an induction on $k$ will complete the proof. 
As in (9.4), we verify by (9.6), (9.7) that

$$
\mathcal{P}^{k} \mathcal{P}^{p^{i-k}} g_{n}^{\#}=0
$$

for $0<k<p^{i}$.

\section{HOMOTOPY GROUPS AND 2-FOLD AND 3-FOLD CYCLIC PRODUCTS OF SPHERES}

10. The problem. Let $K$ be a connected complex. Consider the $q$-fold symmetric product $K^{\Sigma_{q}}$ of $K$. We regularly imbed $K$ in $K^{\Sigma_{q}}$ in the sense of $\$ 3$ with reference point $s_{0} \in K$, and denote by $\iota: \pi_{s}(K) \rightarrow \pi_{s}\left(K^{\Sigma_{q}}\right)$ the usual injection homomorphism between homotopy groups. Let $\alpha \in \pi_{s}(K)$. If $\iota(\alpha)=0$, we shall say that $\alpha$ is killed by $q$-fold symmetrization of $K$. We assert that the behavior of $\alpha$ being killed by symmetrization is independent of the choice of the reference point $s_{0} \in K$ to define the regular imbedding. In fact, given any other $s_{0}^{\prime} \in K$, there is a homotopy equivalence $f: K \rightarrow K$ with $f\left(s_{0}\right)=s_{0}^{\prime}$, and, as was shown in $\S 1, f^{\Sigma_{q}}$ is a homotopy equivalence: $K^{\Sigma_{q}} \rightarrow K^{\Sigma_{q}}$. Also, after imbedding $K$ into $K^{\Sigma_{a}}$ with respect to both the reference points $s_{0}$, $s_{0}^{\prime} \in K$, we see that the partial map $f^{\Sigma_{q}} \mid s_{0}(K)$ is the same as $f$. Homotopy equivalence induces isomorphisms between homotopy groups. Our assertion is thus clear.

We may give also the meaning for the expression that the elements of $\pi_{s}(K)$ are killed in $\Gamma$-products of $K$ in the same way as above with $\Sigma_{q}$ replaced by $\Gamma$. Let $\Gamma \subset \Sigma_{q}$. By the natural map $\eta_{\Gamma \Sigma_{q}}: K^{\Gamma} \rightarrow K^{\Sigma_{q}}(\S 3)$, it is clear that $\alpha \in \pi_{s}(K)$ is killed in $K^{\Sigma_{q}}$ if it is killed in $K^{\Gamma}$.

Let $s_{0} \in K$ be given. We imbed $K^{\Sigma_{q}}$ in $K^{\Sigma_{q+1}}$ as follows. Let $f_{q}: K^{q} \rightarrow K^{q+1}$ be the map sending $\left(x_{1}, x_{2}, \cdots, x_{q}\right)$ to $\left(x_{1}, x_{2}, \cdots, x_{q}, s_{0}\right)$. It is clearly an into-homeomorphism. Form $K^{\Sigma_{q}}$ and $K^{\Sigma_{q+1}}$ respectively over $K^{q}$ and $K^{q+1} \cdot f_{q}$ gives rise naturally to a $\operatorname{map} f_{q}^{\prime}: K^{\Sigma_{q}} \rightarrow K^{\Sigma_{q+1}}$. It is easily seen that $f_{q}^{\prime}$ is an into-homeomorphism. Let us identify $K^{\Sigma_{q}}$ and $f_{q}^{\prime}\left(K^{\Sigma_{q}}\right)$ under $f_{q}^{\prime}$. We have thus the imbedding: $K=K^{\Sigma_{1}} \subset K^{\Sigma_{2}} \subset K^{\Sigma_{3}} \subset \ldots$ Clearly, $\alpha \in \pi_{8}(K)$ is killed by $(q+1)$-fold symmetrization, if it is killed by $q$-fold symmetrization.

We consider the simple case $K=S_{n}$. We have the problem:

How many homotopy groups of $S_{n}$ could be killed by symmetrization?

The results in later sections give that 2-fold symmetrization of $S_{n}$ kills $\pi_{n+1}\left(S_{n}\right), \pi_{n+2}\left(S_{n}\right)$, the 2-primary subgroup of $\pi_{n+3}\left(S_{n}\right)$ for $n \geqq 5$; 3 -fold symmetrization kills the 3 -primary subgroup of $\pi_{n+3}\left(S_{n}\right)$ for $n \geqq 3$. Symmetrization kills all homotopy groups $\pi_{8}\left(S_{2}\right)$ for $s>2$, since the $q$-fold symmetric product of $S_{2}$ is homeomorphic to the complex projective space $M_{2 q}$ of (topological) dimension $2 q$. (Take $S_{2}$ as the extended complex plane and express $M_{2 q}$ by homogeneous coordinates in complex numbers. Define $\phi: S_{2}^{q}$ $\rightarrow M_{2 q}$ by taking $\phi\left(z_{1}, z_{2}, \cdots, z_{q}\right)=\left(a_{0}, a_{1}, \cdots, a_{q}\right)$ if the $z_{i}$ 's are the $n$ roots of the equation $\sum_{i=0}^{q} a_{i} x^{i}=0$. Identifying the inverse image under $\phi$ of every point of $M_{2 q}$ gives a homeomorphism between $S^{\Sigma_{q}}$ and $M_{2 q}$.) It seems true that more and more homotopy groups of spheres are killed by sym- 
metrization. Perhaps one of the reasons might be illustrated by the proof of (13.7), in which we compare the cohomology groups of a symmetric product and those of an Eilenberg-MacLane complex.

Very little is known concerning the homology properties of symmetric products $\left({ }^{2}\right)$. It follows easily from (3.5) and (6.2) that, if $g_{n} \in H^{n}\left(S_{n}, Z\right)$ is the generator and $\mu: H^{n}\left(S_{n}, Z\right) \rightarrow H^{n}\left(S_{n}^{\Sigma}, Z\right)$ is the into-isomorphism (3.2), then, in $S_{n}^{\Sigma}$, we have $\mathcal{P}^{k} \mu\left(g_{n}\right) \neq 0$ for all cyclic reduced powers $\bmod p$ and $p$ prime $\leqq q$ and $2 k \leqq n$.

11. The cellular decomposition of $S_{n} * S_{n}$ of Steenrod. As the integral cohomology groups of $\vartheta_{n p}(n \geqq 2)$ are computed as in (5.4), its integral homology groups can be easily obtained by duality theorems. $\vartheta_{n p}$ is simply connected by (1.4). Thus, following a statement given in later Appendix, there is a finite cellular complex $\left(^{3}\right) \vartheta_{n p}^{\prime}$ of the same homotopy type of $\vartheta_{n p}$, composed of oriented open cells $C$ 's, $D$ 's as follows:

$$
\begin{aligned}
\vartheta_{n p}^{\prime}= & \left(C_{0} \cup C_{n} \cup C_{n+2} \cup C_{n+3} \cup \cdots \cup C_{n p}\right) \\
& \bigcup_{r=2}^{p-1}\left(D_{n r}^{1} \cup D_{n r}^{2} \cup \cdots \cup D_{n r}^{C_{p, r} / p}\right),
\end{aligned}
$$

where $(\alpha)$ the subscript of $C, D$ denotes the dimension of the cell, $(\beta)$ the homotopy boundary $\left({ }^{10}\right)$ of $C_{n+2 k}$ is contained in the $(n+2(k-1))$ th skeleton of $\vartheta_{n p}^{\prime},(\gamma)$ the homotopy boundary of $D_{n r}^{i}$ is contained in the $(n r-2)$ th skeleton of $\vartheta_{n p}^{\prime}$, and $(\delta)$ the homology boundary $\left({ }^{11}\right)$ of $C_{n+2 k+1}$ is $p C_{n+2 k}$.

We do not know apparently the homotopy boundaries of the open cells in (11.1). For $p=2$, an explicit cellular decomposition of $\vartheta_{n p}$ was given by Steenrod $\left.{ }^{12}\right)$. The basic lemma for this is the following (11.2).

(11.2) $S_{n} * S_{n}$ is homeomorphic to the space obtained by first suspending $S_{n-1} * S_{n-1}$ and then adjoining a $2 n$-cell to the suspension.

Let $S_{n}$ be the set in the Euclidean $(n+1)$-space defined by the equation $\sum_{i=1}^{n+1} u_{i}=1 . S_{n}$ is cut by the hyperplane $u_{n+1}=0$ into upper cap $E_{+}$and lower cap $E_{-}$and $S_{n-1}=E_{+} \cap E_{-}$. We shall write $[x, y]=\bar{I}(x, y) \in S_{n} * S_{n}$ for $(x, y)$ $\in S_{n} \times S_{n}$. Let $K$ be the subset of points $[x, y]$ in $S_{n} * S_{n}$ such that $x$ and $y$ have the same latitude in $S_{n}$ relative to $S_{n-1}$. Clearly $K$ is homeomorphic to the suspension of $S_{n-1} * S_{n-1}$. For any point $x$ of $S$, let $\theta(x)$ be its latitude $\left(+\right.$ in $E_{+},-$in $\left.E_{-}\right),-\pi / 2 \leqq \theta(x) \leqq \pi / 2$. Define $\phi: E_{+} \times E_{-} \rightarrow S_{n} * S_{n}$ as follows. Let $x \in E_{+}, y \in E_{-}$. If $\theta(x) \geqq-\theta(y)$, let $y^{\prime}$ be the point on the longitudinal circle of $y$ with latitude $\theta(y)+(\theta(x)+\theta(y))=\theta(x)+2 \theta(y)$ and set $\phi(x, y)=\left[x, y^{\prime}\right]$. If $\theta(x) \leqq-\theta(y)$, let $x^{\prime}$ be the point on the longitudinal circle

(10) See $[14$, p. 221$]$. We call the partial map over the boundary $\vartheta \sigma_{n}$ of the characteristic map $f: \sigma_{n} \rightarrow \bar{e}_{n}$ the homotopy boundary of the open cell $e_{n}$.

(11) See later Appendix.

(12) The remainder of this section are unpublished results of N. E. Steenrod. Thanks are due to him for his permission to include these results here. 
of $x$ with latitude $\theta(x)+(\theta(x)+\theta(y))=2 \theta(x)+\theta(y)$ and set $\phi(x, y)=\left[x^{\prime}, y\right]$. Note that $\theta(x)=-\theta(y)$ implies $\phi(x, y)=[x, y]$ in both cases, so $\phi$ is a (continuous) map.

We show that $\phi$ is onto. Let $[x, y]$ be given. If $\theta(x)=\theta(y)$ and $x, y \in E_{+}$, let $y_{1}$ be the point on the equator of $S_{n}$ having the longitude of $y$. Then $\phi\left(x, y_{1}\right)=[x, y]$; for $\theta\left(y_{1}\right)=0$ implies first case, so $\phi\left(x, y_{1}\right)=\left(x, y_{1}^{\prime}\right)$ where $\theta\left(y^{\prime}\right)=\theta(x)+2 \theta(y)=\theta(x)$, hence $y_{1}^{\prime}=y$. Similarly, if $\theta(x)=\theta(y)$ and $x, y \in E_{-}$, we work on $x$.

Since $[x, y]=[y, x]$ in $S_{n} * S_{n}$, we need consider only the case $\theta(x)>\theta(y)$. We must consider various cases.

$$
\theta(x)+\theta(y) \leqq 0
$$

which implies $\theta(y) \leqq 0$. Solve equation $\theta(x)=2 \theta(x)+\theta(y)$,

$$
\theta\left(x_{1}\right)=[\theta(x)-\theta(y)] / 2,
$$

and let $x_{1}$ have the same longitude as $x$. The $\theta\left(x_{1}\right) \geqq 0$ since $\theta(x) \geqq \theta(y)$ and $\theta\left(x_{1}\right)+\theta(y)=[\theta(x)+\theta(y)] / 2 \leqq 0$, so Case 2 of definition applies and $\phi\left(x_{1}, y\right)$ $=[x, y]$.

$$
\theta(x)+\theta(y) \geqq 0 .
$$

Solve equation $\theta(y)=\theta(x)+2 \theta\left(y_{1}\right)$,

$$
\theta\left(y_{1}\right)=[\theta(y)-\theta(x)] / 2 \leqq 0,
$$

and let $y_{1}$ have the same longitude as $y$. Then Case 1 of definition applies for $\theta\left(y_{1}\right)+\theta(x)=(\theta(y)+\theta(x)) / 2 \geqq 0$, so $\phi\left(x, y_{1}\right)=(x, y)$. Thus, the map $\phi$ is onto.

Now suppose

$$
\phi(x, y)=\phi\left(x_{1}, y_{1}\right), \quad x, x_{1} \in E_{+}, y, y_{1} \in E_{-} .
$$

Suppose first that $\theta(x)+\theta(y) \geqq 0$. Then $\phi(x, y)=\left[x, y^{\prime}\right]$ as in Case 1 of definition, and we have $\theta(x)+\theta\left(y^{\prime}\right)=2[\theta(x)+\theta(y)] \geqq 0$. Now $\theta\left(x_{1}\right)+\theta\left(y_{1}\right)<0$ would lead to $\phi\left(x_{1}, y_{1}\right)=\left(x_{1}{ }^{\prime}, y_{1}\right)$ with $\theta\left(x_{1}^{\prime}\right)+\theta\left(y_{1}\right)=2\left[\theta\left(x_{1}\right)+\theta\left(y_{1}\right)\right]<0$ which contradicts $\phi(x, y)=\phi\left(x_{1}, y_{1}\right)$. So we must have $\theta\left(x_{1}\right)+\theta\left(y_{1}\right) \geqq 0$. Hence $\phi\left(x_{1}, y_{1}\right)$ $=\left(x_{1}, y_{1}^{\prime}\right)$ with $\theta\left(x_{1}\right)+\theta\left(y_{1}^{\prime}\right)=2\left[\theta\left(x_{1}\right)+\theta\left(y_{1}\right)\right]$. Suppose first that $x_{1}=x$, $y_{1}^{\prime}=y^{\prime}$. Then

$$
\theta\left(y^{\prime}\right)=\theta(x)+2 \theta(y), \quad \theta\left(y_{1}^{\prime}\right)=\theta\left(x_{1}\right)+2 \theta\left(y_{1}\right)
$$

imply $\theta(y)=\theta\left(y_{1}\right)$, so we obtain $(x, y)=\left(x_{1}, y_{1}\right)$, or else $x=x_{1}=$ north pole of $S_{n}$ and $y, y_{1}=$ any two points on $S_{n-1}$. Suppose then that $x_{1}=y^{\prime}$ and $x=y_{1}^{\prime}$. By equations above we get $\theta(y)=-\theta\left(y_{1}\right)$. Since both $y, y_{1} \in E_{-}$we must have $\theta(y)=\theta\left(y_{1}\right)=0$. This implies that $(x, y)$ and $\left(x_{1}, y_{1}\right)$ are both in $E_{+} \times S_{n-1}$. Assuming $\theta(x)+\theta(y)<0$ we deduce similarly that $\phi(x, y)=\phi\left(x_{1}, y_{1}\right)$ implies $(x, y)=\left(x_{1}, y_{1}\right)$ or that both points are in $S_{n-1} \times E_{-}$.

To sum up, $\phi: E_{+} \times E_{-} \rightarrow S_{n} * S_{n}$ maps the boundary $E_{+} \times S_{n-1} \cup S_{n-1} \times E_{-}$ 
of $E_{+} \times E_{-}$onto $K \subset S_{n} * S_{n}$ and maps the interior of $E_{+} \times E_{-}$onto $S_{n} * S_{n}-K$ topologically. (11.2) is proved.

Obviously, using (11.2), a cellular decomposition

$$
S_{n} * S_{n}=C_{0} \cup C_{n} \cup C_{n+2} \cup \ldots \cup C_{2 n}
$$

can be constructed by induction on $n$ where the subscript of $C$ denotes the dimension number of the cell. We start with $n=2 . S_{2} * S_{2}$ is the complex projective plane, and we take the desired cellular decomposition of $S_{2} * S_{2}$ as that given in $[10$, p. 310]. Suppose that (11.3) has been already constructed for $S_{n-1} * S_{n-1}$. We decompose $K \subset S_{n} * S_{n}$ first into $K=C_{0} \cup C_{n}$ $\cup C_{n+2} \cup \ldots \cup C_{2 n-1}$ as a homeomorphic image of the suspension of the decomposition of $S_{n-1} * S_{n-1}$ (by the homeomorphism given in above proof) and then adjoin a $2 n$-cell to $K$ by means of the map $\phi$. Clearly, the partial map $\phi \mid S_{n-1} * S_{n-1}$ coincides with the identification map $\bar{I}: S_{n-1} \times S_{n-1} \rightarrow S_{n-1} * S_{n-1}$, and the involution $T$ acting on $S_{n-1} \times S_{n-1}$ preserves or reverses orientation according as $n$ is odd or even. Let the $C_{i}$ 's in (11.3) be oriented arbitrarily. By a consideration of the partial map $\phi \mid\left(E_{+} \times S_{n-1} \cup S_{n-1} \times E_{-}\right)$, we find that, in (11.3), the homology boundary of $C_{2 n}$ is $\pm 2 C_{2 n-1}$ or 0 according as $n$ is odd or even. Thus, by the inductive construction of (11.3) and by some usual property of suspension, we conclude that the homology boundary of $C_{n+j}$ $\left(j \geqq 3\right.$ ) is $\pm 2 C_{n+j-1}$ or 0 according as $j$ is odd or even. (11.3) can be also applied to show that $S q^{i} g_{n}^{\sharp} \neq 0,2 \leqq j \leqq n$. In fact, using (6.1) and the commutativity between $S q^{j}$ and suspension, we have first $S q^{2 k} g_{n}^{\sharp} \neq 0,2 \leqq 2 k \leqq n$. Then, since the Bockstein homomorphism maps $H^{2 k}\left(S_{n} * S_{n}, Z_{2}\right)$ onto $H^{2 k+1}\left(S_{n} * S_{n}, Z_{2}\right)$, we have $S q^{2 k+1} g_{n}^{*} \neq 0,2 \leqq 2 k+1 \leqq n$.

12. $p$-primary subgroups of $\pi_{q}\left(S_{n}\right), n \geqq 3$. It is known that the $p$-primary subgroup $C\left(\pi_{q}\left(S_{n}\right), p\right)$ of $\pi_{q}\left(S_{n}\right), n \geqq 3$, vanishes for $n<q<n+2 p-3$, and $C\left(\pi_{n+2 p-3}\left(S_{n}\right), p\right) \approx Z_{p}$ [5]. Let us give the characteristic property of $C\left(\pi_{n+2 p-3}\left(S_{n}\right), p\right)$ by reduced powers.

Denote by $K\left(S_{n}\right)$ an Eilenberg-MacLane complex, which is cellular, and whose only nonvanishing homotopy group is $\pi_{n}\left(K\left(S_{n}\right)\right)$ and is isomorphic to $\pi_{n}\left(S_{n}\right)$. If $K$ is arbitrary cellular complex whose first nonvanishing homotopy group is $\pi_{n}(K) \approx \pi_{n}\left(S_{n}\right)$, using some standard method we may imbed $K$ into a complex $K\left(S_{n}\right)$ such that the injection homomorphism: $\pi_{n}(K) \rightarrow \pi_{n}\left(K\left(S_{n}\right)\right)$ is an onto-isomorphism. We say then that $K\left(S_{n}\right)$ is an Eilenberg-MacLane complex constructed starting with $K$. Any two complex $K\left(S_{n}\right)$ are of the same homotopy type. Clearly, $H^{n}\left(K\left(S_{n}\right), Z_{p}\right) \approx Z_{p}$. Let $\alpha_{n} \in H^{n}\left(K\left(S_{n}\right), Z_{p}\right)$ be a generator.

(12.1) In $K\left(S_{n}\right)$, we have $\mathbb{P}^{k} \alpha_{n} \neq 0,0 \leqq 2 k \leqq n$.

In fact, it follows from (1.4) and (5.4) that $\pi_{s}\left(\vartheta_{n p}\right)$ vanishes for $1 \leqq s<n$ and $\approx Z$ for $s=n$. Construct $K\left(S_{n}\right)$, starting with $\vartheta_{n p}$. We may assume that $i^{*}\left(\alpha_{n}\right)=g_{n}^{*}$ where $i$ denotes the inclusion $\vartheta_{n p} \subset K\left(S_{n}\right)$. Then (12.1) follows from (6.2) and the commutativity $\mathcal{P}^{k} i^{*}=i^{*} \Phi^{k}$. 
Similarly, we may show that, in $K\left(S_{n}\right), S q^{j} \alpha_{n} \neq 0,2 \leqq j \leqq n$.

We shall write $K\left(S_{n}, p\right)$ for the cellular complex which is the union of the cellular complexes $S_{n}=K_{1}, K_{2}, K_{3}, \cdots$, where $K_{s}$ is constructed by adjoining cells to $K_{s-1}$, by means of maps $f_{j}$ of a $q_{s}$-sphere into $K_{s-1}$ which represent a basis of the $p$-primary subgroup $C\left(\pi_{q}\left(K_{s-1}\right), p\right)$ of $\pi_{q_{s}}\left(K_{s-1}\right)$ with $C\left(\pi_{q_{s}}\left(K_{8-1}\right), p\right)$ nonvanishing and $q_{s}$ least. It is easy to verify by our construction that the $p$-primary subgroup of $\pi_{q}\left(K\left(S_{n}, p\right)\right)$ vanishes for every $q>n$ and $\pi_{n}\left(K\left(S_{n}, p\right)\right) \approx \pi_{n}\left(S_{n}\right)$.

Write

$$
E_{m}=\text { an oriented } m \text {-cell( }\left(^{8}\right) .
$$

Denote by $E_{f}$ the cellular complex constructed by adjoining $E_{q+1}$ to $S_{n}$, by means of a map $f: \partial E_{q+1} \rightarrow S_{n}, q>n$. Clearly, $H^{k}\left(E_{f}, Z_{p}\right) \approx Z_{p}$ for $k=n, q+1$ and vanishes for other $s$. Let $S_{n}$ be oriented and let $\beta_{n} \in H^{n}\left(E_{f}, Z_{p}\right)$ be the generator represented by $S_{n}$, and write

$$
e_{f} \in \pi_{q}\left(S_{n}\right) \text { for the element represented by } f \text {. }
$$

Let $q=n+2 k(p-1)-1$. We observe that $\mathcal{P}^{k} \beta_{n} \in H^{n+2 k(p-1)}\left(E_{f}, Z_{p}\right)$ gives rise to an element $e_{f}^{\prime} \in H^{n+2 k(p-1)}\left(E_{n+2 k(p-1)}, \partial E_{n+2 k(p-1)} ; Z_{p}\right)$ by identifying these two groups in a natural manner. For $n \geqq 3$, let us define

$$
\psi: \pi_{n+2 k(p-1)-1}\left(S_{n}\right) \rightarrow H^{n+2 k(p-1)}\left(E_{n+2 k(p-1)}, \partial E_{n+2 k(p-1)} ; Z_{p}\right)
$$

by taking $\psi\left(e_{f}\right)=e_{f}^{\prime} \cdot \psi$ is then a homomorphism $\left({ }^{13}\right)$.

(12.3) The homomorphism $\psi$ with $k=1$ maps $C\left(\pi_{n+2 p-3}\left(S_{n}\right), p\right)$ isomorphically onto $H^{n+2(p-1)}\left(E_{n+2(p-1)}, \partial E_{n+2(p-1)} ; Z_{p}\right)(n \geqq 3)$. If $e \in \pi_{n+2 p-3}\left(S_{n}\right)$ is of finite order prime to $p$, then $\psi(e)=0$.

Consider first $n=3$. Since $C\left(\pi_{q}\left(S_{3}\right), p\right)=0,3<q<2 p$, in the complex $K\left(S_{3}, p\right)$ there is no cell of dimension greater than 3 and less than $2 p+1$, and since $C\left(\pi_{2 p}\left(S_{3}\right), p\right) \approx Z_{p}$ there is exactly one $(2 p+1)$-cell with homotopy boundary $f: \partial E_{2 p+1} \rightarrow S_{3}$ representing a nonzero element of $C\left(\pi_{2 p}\left(S_{3}\right), p\right)$, and $K\left(S_{3}, p\right)$ contains thus $E_{f}$. Construct the cellular complex $K\left(S_{3}\right)$, starting with $K\left(S_{3}, p\right)$. Then, by the exactness of the homotopy sequence of the pair $\left(K\left(S_{3}\right), K\left(S_{3}, p\right)\right)$, we have that the $p$-primary subgroup of $\pi_{q}\left(K\left(S_{3}\right), K\left(S_{3}, p\right)\right)$ vanishes for $1<q \leqq 2 p+1$. Also, $\pi_{q}\left(K\left(S_{3}\right), K\left(S_{3}, p\right)\right)$ has no element of order infinity for $1<q \leqq 2 p+1$. It follows therefore from the generalized Hurewicz theorem as given by J. Moore [5] that $H^{2 p+1}\left(K\left(S_{3}\right), K\left(S_{3}, p\right) ; Z_{p}\right)=0$. Then, using the exactness of the cohomology sequence of the pair $\left(K\left(S_{3}\right), K\left(S_{3}, p\right)\right)$, (12.1) gives that, in $E_{f}, \Phi^{1} \beta_{n} \neq 0$. Hence, $\psi$ is onto for $n=3$. Using suspension, we see that $\psi$ maps $C\left(\pi_{n+2 p-3}\left(S_{n}\right), p\right)$ on to $H^{n+2(p-1)}\left(E_{n+2(p-1)}, \partial E_{n+2(p-1)} ; Z_{p}\right)$. It is obvious that $\psi$ maps an element of $\pi_{n+2 p-3}\left(S_{n}\right)$ of finite order prime to $p$ into 0 .

${ }^{(13)}$ It follows from some theorem of Adem that $\theta$ is 0 if $k$ is not a power of $p$. For the case $p=2$, see [1]. 
13. On homotopy groups of 2-fold and 3-fold cyclic products of spheres. We regularly imbed $S_{n}$ in $\vartheta_{n p}$ and write

$$
S_{n} \subset \vartheta_{n p}
$$

Let $S_{n}$ be oriented and let $g_{n}^{*}$ be so chosen that the projection homomorphism: $H^{n}\left(\vartheta_{n p}, Z\right) \rightarrow H^{n}\left(S_{n}, Z\right)$ maps $g_{n}^{*}$ into the generator of $H^{n}\left(S_{n}, Z\right)$ represented by $S_{n}$. Write

$$
\xi=\text { the injection homomorphism: } H^{s}\left(\vartheta_{n p}, S_{n} ; G\right) \rightarrow H^{s}\left(\vartheta_{n p}, G\right),
$$

where $G$ is an arbitrary coefficient group. $\xi$ is an isomorphism for $s>n$. As in (12.2), we define

$$
\psi: \pi_{3}\left(S_{2}\right) \rightarrow H^{4}\left(E_{4}, \partial E_{4} ; Z\right)
$$

by taking now $\psi\left(e_{f}\right)=e_{f}^{\prime}$ with $e_{f}^{\prime}$ corresponding to $\beta_{2}^{2} \in H^{4}\left(E_{f}, Z\right) . \psi$ is an onto-isomorphism. For if $f=f_{0}: \partial E_{4} \rightarrow S_{2}$ is a map with Hopf invariant 1, we have $\psi\left(e_{f_{0}}\right) \in H^{4}\left(E_{4}, \partial E_{4} ; Z\right)$ represented by the oriented cell $E_{4}[10$, p. 311]. Some usual arguments give then $\psi\left(t e_{f_{0}}\right)=t \psi\left(e_{f_{0}}\right)$ for any integer $t$.

(13.3) The $(n+1)$ th homotopy group of $S_{n} * S_{n}$ vanishes $(n \geqq 2)$. Thus, every map $f: \partial E_{n+2} \rightarrow S_{n}$ extends in $S_{n} * S_{n}$ over $E_{n+2}$. Moreover, if $F$ is such an extension of $f$, we have:

$$
\begin{array}{ll}
\psi\left(e_{f}\right)=F^{*} \xi^{-1}\left(g_{2}^{* 2}\right) \in H^{4}\left(E_{4}, \partial E_{4} ; Z\right), & n=2, \\
\psi\left(e_{f}\right)=F^{*} \xi^{-1} S q^{2} g_{n}^{*} \in H^{4}\left(E_{n+2}, \partial E_{n+2} ; Z_{2}\right), & n \geqq 3,
\end{array}
$$

where $\psi$ is the isomorphism (13.2) or (12.2) with $k=1, p=2$, according as $n=2$ or $n \geqq 3\left({ }^{1}\right)$.

In fact, $S_{2} * S_{2}$ is the complex projective plane, so $\pi_{3}\left(S_{2} * S_{2}\right)=0$. For $n \geqq 3$, we observe that the subcomplex $C_{0} \cup C_{n} \cup C_{n+2}$ of $S_{n} * S_{n}$ with respect to the cellular decomposition (11.3) is homeomorphic to the complex $M_{n+2}$ considered by Steenrod [10, p. 311], and by arguments there, we verify that the boundary homomorphism

$$
\partial: \pi_{n+2}\left(S_{n} * S_{n} ; W_{n}\right) \rightarrow \pi_{n+1}\left(W_{n}\right)
$$

is onto, where $W_{n}=C_{0} \cup C_{n}$ is an $n$-sphere. Clearly, $H_{s}\left(S_{n} * S_{n}, W_{n} ; Z\right)=0$ for $s<n+2$ and hence, by (1.4), the Hurewicz isomorphism gives $\pi_{n+1}\left(S_{n} * S_{n} ; W_{n}\right)$ $=0$. So, by the exactness of the homotopy sequence of the pair $\left(S_{n} * S_{n}, W_{n}\right)$, we have $\pi_{n+1}\left(S_{n} * S_{n}\right)=0$.

To prove (13.4), we need merely identify $\partial E_{n+2}$ with $S_{n}$ by the map $f$ and use the definition of $\psi$. Then, $F$ gives rise naturally to a map $F^{\prime}: E_{f} \rightarrow S_{n}$ $* S_{n}$. Since the homomorphism induced by a map preserves cup product and square operations, we deduce thus (13.4) easily.

(13.6) The $(n+2)$ th homotopy groups of $S_{n} * S_{n}$ vanishes $(n \geqq 2)\left({ }^{1}\right)$.

This can be proved by some arguments similar to those given before. For 
$n=2$, this follows from the fact that the fourth homotopy group of the complex projective plane vanishes. Hence, every map: $S_{4} \rightarrow W_{2}$ can be deformed in $M_{4}$ into a point. Suppose inductively that a map $f: S_{n+2} \rightarrow W_{n}$ for a certain $n \geqq 2$ can be deformed in $M_{n+2}$ into a point $s$. Note that the suspension of the pair $\left(M_{n+2}, W_{n}\right)$ is the same as the pair $\left(M_{n+3}, W_{n+1}\right)$ (up to a homeomorphism) and the suspension of $\pi_{n+2}\left(W_{n}\right)$ is $\pi_{n+3}\left(W_{n+1}\right)$ (for $W_{n}$ and $W_{n+1}$ are spheres). It follows that the suspension of $f$ in $M_{n+3}$ can be deformed into a broken line in $M_{n+3}$, and hence every map: $S_{n+3} \rightarrow W_{n+1}$ can be deformed into a point. This gives that the injection homomorphism: $\pi_{n+2}\left(W_{n}\right)$ $\rightarrow \pi_{n+2}\left(S_{n} * S_{n}\right)$ is trivial.

Let $n \geqq 3$. As in the cellular decomposition (11.3), the homology boundary of $C_{n+3}$ is $\pm 2 C_{n+2}$, so $H_{n+2}\left(S_{n} * S_{n}, W_{n} ; Z\right) \approx Z_{2}$ and as before we deduce $\pi_{n+2}\left(S_{n} * S_{n}, W_{n}\right) \approx Z_{2}$. This gives that the boundary homomorphism (13.5) is an onto-isomorphism. We get thus (13.6) easily.

In (12.2), let $n \geqq 4, k=2, p=2$. It was known that the 2-primary subgroup of $\pi_{n+3}\left(S_{n}\right) \approx Z_{8}$ for $n \geqq 5$ [4]. If $f$ is a map: $S_{7} \rightarrow S_{4}$ with Hopf invariant 1 , $S q^{4} \beta_{4}=$ the generator of $H^{8}\left(E_{f}, Z_{2}\right)$ [1]. So $\psi$ is onto for $n=4$. The suspension gives that $\psi$ is onto for $n \geqq 5$, and hence $\pi_{n+3}\left(S_{n}\right) / 2 \pi_{n+3}\left(S_{n}\right)$ is characterized by $S q^{4}$ for $n \geqq 5$.

(13.7) The 2-primary subgroup $C\left(\pi_{n+3}\left(S_{n} * S_{n}\right), 2\right)$ of $\pi_{n+3}\left(S_{n} * S_{n}\right)$ vanishes for $n \geqq 5$. Thus every map $f: \partial E_{n+4} \rightarrow S_{n}$ which represents an element of $C\left(\pi_{n+3}\left(S_{n} * S_{n}\right), 2\right), n \geqq 5$, extends in $S_{n} * S_{n}$ over $E_{n+4}$. Moreover, if $F$ is such an extension, we have:

$$
\psi\left(e_{f}\right)=F^{*} \xi^{-1}\left(S q^{4} g_{n}^{*}\right) \in H^{n+4}\left(E_{n+4}, \partial E_{n+4} ; Z_{2}\right),
$$

where $\psi$ is the homomorphism (12.2) with $k=2, p=2$.

To prove this, we construct the Eilenberg-MacLane complex $K\left(S_{n}\right)$, starting with $S_{n} * S_{n}$. Consider the homomorphism $i_{*}$ between homology groups and also between homotopy groups induced by the inclusion $S_{n} * S_{n}$ $\subset K\left(S_{n}\right)$ and the dual homomorphism $i^{*}$ between cohomology groups. Let $n \geqq 5$. We have that $H_{8}\left(S_{n} * S_{n}, Z_{2}\right)=H_{8}\left(K\left(S_{n}\right), Z_{2}\right)=0$ for $1 \leqq s \leqq n-1$ and $s=n+1, H_{s}\left(S_{n} * S_{n}, Z_{2}\right) \approx Z_{2} \approx H_{s}\left(K\left(S_{n}\right), Z_{2}\right)$ for $n+2 \leqq s \leqq n+4$ [2, pp. 661$662]{ }^{(14)}$. Obviously, $i_{*}: H_{n}\left(S_{n} * S_{n}, Z\right) \approx H_{n}\left(K\left(S_{n}\right), Z\right)$. Then, since, in $S_{n} * S_{n}$, $S q^{i} g_{n}^{*}=0$ for $2 \leqq j \leqq n$, we see easily by the commutativity $i^{*} S q^{i}=S q^{i} i^{*}$ that $i_{*}: H_{s}\left(S_{n} * S_{n}, Z_{2}\right) \rightarrow H_{s}\left(K\left(S_{n}\right), Z_{2}\right)$ is an into-isomorphism. Thus, $H_{s}\left(K\left(S_{n}\right)\right.$, $\left.S_{n} * S_{n} ; Z_{2}\right)=0$ for $1 \leqq s \leqq n+4$ and by the generalized relative Hurewicz theorem [5], $\pi_{s}\left(K\left(S_{n}\right), S_{n} * S_{n}\right) \otimes Z_{2}=0$ for $1 \leqq s \leqq n+4$. This leads to that $i_{*}$ :

$$
C\left(\pi_{s}\left(S_{n} * S_{n}\right), 2\right) \approx C\left(\pi_{s}\left(K\left(S_{n}\right), 2\right)=0\right.
$$$$
\text { for } n<s \leqq n+3 \text {. }
$$

Now, let $f$ be a map: $\partial E_{n+4} \rightarrow S_{n}$, representing the generator of

${ }^{(14)}$ In [2, p. 662] only integral homology groups $A_{i}(Z)$ are given explicitly. However, we may then determine $A^{i}\left(\pi_{n}\left(S_{n}\right), Z_{2}\right)$ from $A_{i}(Z)$ by usual arguments in universal coefficient theorems and duality theorems. 
$C\left(\pi_{n+3}\left(S_{n}\right), 2\right), n \geqq 5$. $f$ extends over $E_{n+4}$ in $S_{n} * S_{n}$. If $F$ is such an extension, then, since $\psi$ is an onto-homomorphism (as was shown previously), we verify easily (13.8). The proof can be then completed by usual arguments.

(13.9) Every map $f: \partial E_{n+4} \rightarrow S_{n}$ extends in $\vartheta_{n 3}$ over $E_{n+4}$ if it represents an element of $C\left(\pi_{n+3},\left(S_{n}\right), 3\right), n \geqq 3$. If $F$ is such an extension, we have

$$
\psi\left(e_{f}\right)=F^{*} \xi^{-1} \mathbb{P}^{1} g_{n}^{*} \in H^{n+4}\left(E_{n+4}, \partial E_{n+4} ; Z_{3}\right)
$$

where $\psi$ is the homomorphism (12.2) with $k=1, p=3$, and the cyclic reduced power $P^{1}$ is defined for $p=3$.

To prove (13.9), we consider the complex $\vartheta_{n 3}^{\prime}$ and its cellular decomposition in (11.1). $C_{n}$ represents the generator $g_{n}^{\prime}$ of the infinite cyclic group $H^{n}\left(\vartheta_{n 3}^{\prime}, Z\right)$; $C_{n+2}$ and $C_{n+4}$ represent respectively elements of $H_{n+2}\left(\vartheta_{n 3}^{\prime}, Z\right), H_{n+4}\left(\vartheta_{n 3}^{\prime}, Z\right)$ of order 3.

Write $S_{n}^{\prime}=C_{0} \cup C_{n}$, which is an $n$-sphere. Let $h_{n+1}: \partial E_{n+2} \rightarrow S_{n}^{\prime}$ denote the homotopy boundary of $C_{n+2}\left({ }^{10}\right)$. We see easily that $\pi_{n+2}\left(\vartheta_{n 3}^{\prime}, S_{n}^{\prime}\right) \approx Z_{3}$. But the 3-primary subgroup of $\pi_{n+1}\left(S_{n}^{\prime}\right)$ vanishes; so $h_{n+1} \simeq 0$ in $S_{n}^{\prime}$. Therefore, by usual arguments in homotopy theory, we shall assume that the complex $\vartheta_{n 3}^{\prime}$ is such that the homotopy boundary of $C_{n+2}$ in $S_{n}^{\prime}$ is null; in other words, $C_{0} \cup C_{n} \cup C_{n+2}$ is the union $S_{n}^{\prime} \bigvee S_{n+2}^{\prime}$ of an $n$-sphere and an $(n+2)$-sphere with one point in common.

Let $h_{n+3}: \partial E_{n+4} \rightarrow S_{n}^{\prime} \bigvee S_{n+2}^{\prime}$ denote the homotopy boundary of $C_{n+4}$. Let $e_{n+3} \in \pi_{n+3}\left(K_{n+3}\right)$ be represented by $h_{n+3}$ where $K_{n+3}$ denotes the $(n+3)$ skeleton of $\vartheta_{n 3}^{\prime}$. Then, since $C_{n+4}$ represents the element of $H_{n+4}\left(\vartheta_{n 3}^{\prime}, Z\right)$ of order 3 , it follows easily that $3 e_{n+3}=0$. But $e_{n+3} \neq 0$ for $\mathcal{P}^{1} g_{n}^{\prime} \neq 0$ (by (6.2)). Therefore $2 e_{n+3}$ has a representative map with range contained in $S_{n}^{\prime}$ since $\pi_{n+3}\left(S_{n+2}^{\prime}\right) \approx Z_{2}$. Let $a_{n+3} \in \pi_{n+3}\left(S_{n}^{\prime}\right)$ be represented by this map. Then, it is clear that $a_{n+3} \neq 0$, any representative map of $a_{n+3}$ is null homotopic in $\vartheta_{n 3}^{\prime}$, and $a_{n+3}$ generates $C\left(\pi_{n+3}\left(S_{n}^{\prime}\right), 3\right.$ ) (since the injection homomorphism: $\pi_{n+3}\left(S_{n}^{\prime}\right) \rightarrow \pi_{n+3}\left(S_{n}^{\prime} \bigvee S_{n+2}^{\prime}\right)$ is an into-isomorphism). It follows that every map: $\partial E_{n+4} \rightarrow S_{n}^{\prime}$ representing an element of $C\left(\pi_{n+3}\left(S_{n}^{\prime}\right), 3\right)$ extends in $\vartheta_{n 3}^{\prime}$ over $E_{n+4}$.

Now, since $\vartheta_{n 3}$ and $\vartheta_{n 3}^{\prime}$ have the same homotopy type, we see easily that every map $f: \partial E_{n+1} \rightarrow S_{n}$ representing an element of $C\left(\pi_{n+3}\left(S_{n}\right), 3\right)$ extends in $\vartheta_{n 3}$ over $E_{n+4}$. If $F$ is such an extension, using (12.3) with $p=3$, the technique which identifies $\partial E_{n+4}$ and $S_{n}$ by $f$ will lead to a proof of (13.10).

14. On secondary obstructions. The problem of the secondary obstruction of maps of a complex into a complex $K$ was solved for the case that $K$ is an $n$-sphere by Steenrod [10]. A generalization to the case that $K$ is $(n-1)$ connected, $n \geqq 2$, was given by J. H. C. Whitehead [16]. We shall show here how the 2 -fold symmetric product of $K$ is related to this extension problem. (Our main purpose is to establish the proposition (14.6) for applications to the study of secondary obstructions of fiber bundles.) 
Throughout this section, we denote by $K$ an $(n-1)$-connected finite cellular complex, $n \geqq 2$, with $s$ th skeleton $K_{s}, X$ an arbitrary cellular complex with sth skeleton $X_{s}$ and $A$ a subcomplex of $X$. Let us recall from $[15 ; 16]$ some properties of the Pontrjagin square and a square operation considered by J. H. C. Whitehead. Let $\omega: S_{n+1} \rightarrow S_{n}$ be a fixed map between oriented spheres which represents the generator of $\pi_{n+1}\left(S_{n}\right)$, with Hopf invariant 1 in case $n=2$. Denote by $\Gamma_{n+1}(K)$ the subgroup of $\pi_{n+1}\left(K_{n+1}\right)$ of elements represented by maps $\omega f: S_{n+1} \rightarrow K_{n+1}$ where $f$ is a map: $S_{n} \rightarrow K_{n+1} . \Gamma_{n+1}(K)$ is an invariant of $K[15]$. The operation: $H^{n}\left(X, A ; \pi_{n}(K)\right) \rightarrow H^{n+2}\left(X, A ; \Gamma_{n+1}(K)\right)$ which is the Pontrjagin square $\mathfrak{p}_{1}$ for $n=2$ and is the square operation $\mathcal{g}^{2}$ for $n \geqq 3$ was considered and studied in $[15 ; 16]$. For convenience, we shall write $S q_{w}^{2}$ for this operation in both cases $n=2$ and $n \geqq 3 . S q_{w}^{2}$ possesses the following properties (14.1) and (14.2).

(14.1) Let $\phi$ be a map of $(X, A)$ into a pair $\left(X^{\prime}, A^{\prime}\right)$ where $X^{\prime}$ is a cellular complex and $A^{\prime}$ a subcomplex of $X^{\prime}$. Then $\phi^{*} S q_{w}^{2}=S q_{w}^{2} \phi^{*}$.

(14.2) Let $h$ be a map of $K^{\prime}$ into $K$ where $K^{\prime}$ is an $(n-1)$-connected finite cellular complex, and denote again by $h$ the homomorphism: $\pi_{s}\left(K^{\prime}\right) \rightarrow \pi_{s}(K)$, $\Gamma_{n+1}\left(K^{\prime}\right) \rightarrow \Gamma_{n+1}(K)$ induced by $h$. Then, in $(X, A), \bar{h} S q_{w}^{2}=S q_{w}^{2} \bar{h}$ where $h$ are the homomorphisms between cohomology groups (namely, $\bar{h}: H^{n}\left(X, A ; \pi_{n}\left(K^{\prime}\right)\right.$ ) $\left.\rightarrow H^{n}\left(X, A ; \pi_{n}(K)\right), H^{n+2}\left(X, A ; \Gamma_{n+1}\left(K^{\prime}\right)\right) \rightarrow H^{n+2}\left(X, A ; \Gamma_{n+1}(K)\right)\right)$ induced by $h$.

The inclusion map: $K_{n+1} \rightarrow K$ gives naturally a homomorphism $\nu: \Gamma_{n+1}(K)$ $\rightarrow \pi_{n+1}(K)$. Let us write $S q_{w}^{\prime 2}=\bar{\nu} S q_{w}^{2}: H^{n}\left(X, A ; \pi_{n}(K)\right) \rightarrow H^{n+2}\left(X, A ; \Gamma_{n+1}(K)\right)$ where $\bar{\nu}$ is the homomorphism: $H^{n+2}\left(X, A ; \Gamma_{n+1}(K)\right) \rightarrow H^{n+2}\left(X, A ; \pi_{n+1}(K)\right)$ induced by $\nu$. We see easily that (14.1) and (14.2) still hold if we replace $S q_{w}^{2}$ by $S q_{w}^{\prime 2}$. In (14.2) if $h$ is a homotopy equivalence, then $h: \Gamma_{n+1}\left(K^{\prime}\right)$ $\approx \Gamma_{n+1}(K)$.

For simplicity, we shall write $\pi_{s}=\pi_{s}(K), \Gamma_{n+1}=\Gamma_{n+1}(K)$, throughout the rest of this section and denote by $\kappa_{n}$ the primary obstruction to contracting $K$, which is a cohomology class $\in H^{n}\left(K, \pi_{n}\right)\left({ }^{15}\right)$.

(14.3) In $K$, we have $S q_{w}^{\prime 2}\left(K_{n}\right)=0$.

For an open cell $C$ in a cellular complex, we write for simplicity $\partial^{\sharp} C$ for the homotopy boundary $\left({ }^{10}\right)$ of $C$. By the properties of $S q_{w}^{\prime 2}$ recalled above we may prove (14.3) by considering any finite cellular complex $K^{\prime}$ which has the same homotopy of $K$, instead of $K$. Thus, by a statement.given in the Appendix we may assume without loss of generality that $K$ is composed of oriented open $k$-cells $C_{k}$ 's, etc., with

$$
\begin{aligned}
K_{n+2}=C_{0} & \cup\left(C_{n}^{1} \cup C_{n}^{2} \cup \ldots \cup C_{n}^{r}\right) \\
& \cup\left(C_{n+1}^{1} \cup C_{n+1}^{2} \cup \ldots \cup C_{n+1}^{s} \cup C_{n+1}^{8+1} \cup \ldots \cup C_{n+1}^{8^{\prime}}\right) \\
& \cup\left(C_{n+2}^{1} \cup C_{n+2}^{2} \cup \ldots \cup C_{n+2}^{t} \cup C_{n+2}^{t+1} \cup \ldots \cup C_{n+2}^{t^{\prime}}\right),
\end{aligned}
$$

(15) See N. E. Steenrod, Topology of fiber bundles, Princeton University Press, 1951, p. 187. 
where $(\alpha) \partial^{\ddagger} C_{n+1}^{i} \subset C_{0} \cup C_{n}^{i}$ for $1 \leqq i \leqq s$ (with $s \leqq r$ ) and is not null homotopic in $C_{0} \cup C_{n}^{i},(\beta) \partial^{*} C_{n+1}^{i} \subset C_{0}$ for $s+1 \leqq i \leqq s^{\prime},(\gamma) \partial^{\sharp} C_{n+2}^{t} \subset K_{n} \cup C_{n+1}^{s+i}$ for $1 \leqq i \leqq t$ (with $s+t \leqq s^{\prime}$ ) and $(\delta) \partial^{*} C_{n+2}^{i} \subset K_{n}$ for $t+1 \leqq i \leqq t^{\prime}$. Since $K_{n}$ is the union of $r$ $n$-spheres contacting at one point, namely $C_{0}$, and $C_{0} \cup C_{n+1}^{3}$ for $s+1 \leqq j \leqq s^{\prime}$ is an $(n+1)$-sphere, $\partial^{\sharp} C_{n+2}^{i}$ for $1 \leqq i \leqq t$ is homotopic in $K_{n} \cup C_{n+1}^{s+i}$ to a map $f_{i}: S_{n+1} \rightarrow K_{n} \cup C_{n+1}^{s+1}$ which maps a hemisphere $E_{n+1}^{i+}$ in $S_{n+1}$ into $K_{n}$ and maps the complementary hemisphere $E_{n+1}^{i-}$ into $C_{0} \cup C_{n+1}^{s+1}$ and $f_{i}\left(\partial^{A} E_{n+1}^{i+}\right)=f_{i}\left(\partial^{\sharp} E_{n+1}^{i-1}\right)$ $=C_{0}$.

We shall consider the secondary boundary homomorphism $\alpha: C_{n+2}(K, Z)$ $\rightarrow \Gamma_{n+1}$ for $K$ as given in [16, p. 71]. We assert that $\nu \alpha: C_{n+2}(K, Z) \rightarrow \pi_{n+1}$, regarded as a cochain with coefficient group $\pi_{n+1}$, is cohomologous to 0 in $K$. In fact, it is easy to see that $\nu \alpha$ has value 0 over $C_{n+2}^{t+1}, C_{n+2}^{t+2}, \cdots, C_{n+2}^{t \prime}$ and $\nu \alpha\left(C_{n+2}^{t}\right)$ for $1 \leqq i \leqq t$ is the element of $\pi_{n+1}$ represented by the map $f_{i}:\left(E_{n+1}^{i}, \partial E_{n+1}^{i-1}\right) \rightarrow\left(C_{0} \cup C_{n+1}^{s+i}, C_{0}\right)$. Regarding $C_{0} \cup C_{n+1}^{s+1}$ as an oriented sphere with orientation given by that of $C_{n+1}^{s+z}$, let $n_{i}$ be the degree of this latter map. Then, $\nu \alpha\left(C_{n+2}^{i}\right), 1 \leqq i \leqq t$, is $n_{i} a^{i}$ where $a^{i}$ is the element of $\pi_{n+1}$ represented by the identity map of $C_{0} \cup C_{n+1}^{s+1}$ into $K$. Let now $u_{n+1}=\sum_{1 \leqq i \leqq t} a^{i} C_{n+1}^{s+4}$, which is an $(n+1)$-cochain of $K$ with coefficient group $\pi_{n+1}$. It is readily seen that $\delta u_{n+1}=\nu \alpha$. We use then the relation $\{\alpha\}=S q_{w}^{2}\left(\kappa_{n}\right)$ in $[16$, p. 79] to complete the proof of (14.3).

We shall write $E_{n+2}$ for an oriented $(n+2)$-cell with boundary = the oriented sphere $S_{n+1}$. We regularly imbed $S_{n}$ into $S_{n} * S_{n}$ and $K$ into $K * K$ in the sense of $\S 3$, and write

$$
S_{n} \subset S_{n} * S_{n}, \quad K \subset K * K .
$$

(14.5) Let $K=S_{n}(n \geqq 2)$. Then, by (13.3), $\omega: S_{n+1} \rightarrow S_{n}$ extends in $S_{n} * S_{n}$ over $E_{n+2}$. If $h$ is such an extension, we have that $h^{*} \xi^{-1} S q_{w}^{\prime 2} \mu\left(\kappa_{n}\right)$ $\in H^{n+2}\left(E_{n+2}, S_{n+1} ; \pi_{n+1}\left(S_{n}\right)\right)$ is represented by the cochain $[\omega] E_{n+2}$ where $[\omega]$ is the element of $\pi_{n+1}\left(S_{n}\right)$ represented by $\omega, \xi$ is the isomorphism (13.1), and $\mu$ is the into-isomorphism: $H^{n}\left(S_{n}, \pi_{n}\left(S_{n}\right)\right) \rightarrow H^{n}\left(S_{n} * S_{n}, \pi_{n}\left(S_{n}\right)\right)$.

In the case $K=S_{n}, S q_{w}^{\prime 2}=S q_{w v}^{2}$. We may deduce easily (14.5) from (13.4)(16).

Let us write the cohomology sequence of the pair $(K * K, K)$ with coefficient group $G$ as

$$
\begin{aligned}
\cdots \rightarrow H^{s}(K * K, K ; G) & \stackrel{\xi}{\rightarrow} H^{s}(K * K, G) \stackrel{\eta}{\rightarrow} H^{s}(K, G) \\
& \stackrel{\delta}{\rightarrow} H^{s+1}(K * K, K ; G) \rightarrow \cdots .
\end{aligned}
$$

It follows from the exactness of this cohomology sequence and (14.3) that there exists a cohomology class $u_{n+2} \in H^{n+2}\left(K * K, K ; \pi_{n+1}\right)$ such that $\xi\left(u_{n+2}\right)$

(16) Here, the identity $2 p_{1} u=u \cup u$ in $[16$, p. 76] is useful for our purpose. Note that the cup product $u \cup u$ in this identity is defined in terms of the pairing $g \cdot g^{\prime}=$ the J. H. C. Whitehead product $\left[g, g^{\prime}\right]$. See also the footnote in $[16$, p. 76$]$. 
$=S q_{w}^{2} \mu\left(K_{n}\right)$ where $\mu$ is the into-isomorphism: $H^{n}\left(K, \pi_{n}\right) \rightarrow H^{n}\left(K * K, \pi_{n}\right)$ given in (3.2).

(14.6) Let $u_{n+2} \in H^{n+2}\left(K * K, K ; \pi_{n+2}\right)$ be such that $\xi\left(u_{n+2}\right)=S q_{w}^{\prime 2} \mu\left(\kappa_{n}\right)$. If $f: S_{n+1} \rightarrow K$ is a map which represents an element of $\Gamma_{n+1}$, then $f$ extends in $K * K$ over $E_{n+2}$. Moreover, if $F$ is such an extension, we have that $F^{*}\left(u_{n+2}\right)$ $\in H^{n+2}\left(E_{n+2}, S_{n+1} ; \pi_{n+1}\right)$ is represented by the cochain $[f] E_{n+2}$ where $[f]$ denotes the element of $\pi_{n+1}$ represented by $f$.

In fact, by replacing a map: $S_{n+1} \rightarrow K$ homotopic to $f$ if necessary, we may assume that $f=f^{\prime} \omega$ where $f^{\prime}$ is a map: $S_{n} \rightarrow K$. We may assume also that $f^{\prime}$ maps the reference point in $S_{n}$ used in the regular imbedding of $S_{n}$ in $S_{n} * S_{n}$ to the reference point in $K$ used in the regular imbedding of $K$ in $K * K$. It follows that $g=f^{\prime} * f^{\prime}$ is a map: $\left(S_{n} * S_{n}, S_{n}\right) \rightarrow(K * K, K)$ such that $g \mid S_{n}=f^{\prime}$. Now, $\omega$ has an extension $h: E_{n+2} \rightarrow S_{n} * S_{n}$, so $F_{0}=g h: E_{n+2} \rightarrow K * K$ is a desired extension of $f$. Since $F_{0}^{*}\left(u_{n+2}\right)=h^{*} g^{*}\left(u_{n+2}\right)$ with $g^{*}: H^{n+2}\left(K * K, K ; \pi_{n+1}\right)$ $\rightarrow H^{n+2}\left(S_{n} * S_{n}, S_{n} ; \pi_{n+1}\right), h^{*}: H^{n+2}\left(S_{n} * S_{n}, S_{n} ; \pi_{n+1}\right) \rightarrow H^{n+2}\left(E_{n+2}, S_{n+1} ; \pi_{n+1}\right)$, using (14.5) and (14.2) for $S q_{w}^{\prime 2}$ we verify that $F_{0}^{*}\left(u_{n+2}\right)$ is represented by $[f] E_{n+2}$. Finally, let $F$ be an arbitrary extension of $f$ in $K * K$ over $E_{n+2}$. We assert that $F^{*}\left(u_{n+2}\right)=F_{0}^{*}\left(u_{n+2}\right)$. In fact, let $E_{\omega}$ be the cellular complex with $n$th skeleton $S_{n}$, constructed by adjoining $E_{n+2}$ to $S_{n}$ by means of the map $\omega$. Then, $F$ and $F_{0}$ give rise naturally to maps $F^{\prime}, F_{0}^{\prime}: E_{\omega} \rightarrow K$ such that $F^{\prime} \mid S_{n}$ $=f=F_{0}^{\prime} \mid S_{n}$, and hence $F^{\prime *} S q_{w}^{\prime 2} \mu\left(K_{n}\right)=F_{0}^{\prime *} S q^{\prime 2} \mu\left(\kappa_{n}\right) \quad\left(=S q^{\prime 2} f^{*}\left(\kappa_{n}\right)\right.$, by (3.6) and (14.1) for $\left.S q_{w}^{\prime 2}\right)$. Our assertion follows then from the fact that $H^{n+2}\left(E_{\omega}, \pi_{n+1}\right)$ and $H^{n+2}\left(E_{n+2}, S_{n} ; \pi_{n+1}\right)$ are isomorphic in a natural manner. This completes the proof of (14.6).

Finally, let us give the following example which shows how to use (14.6) in the extension problem straightforwardly.

(14.7) Let $f$ be a map: $X_{n+1} \rightarrow K$ with $X=a n(n+2)$-dimensional simplicial complex. Then, the secondary obstruction

$$
Z^{n+2}(f) \in H^{n+2}\left(X, \pi_{n+1}\right)
$$

is given by

$$
Z^{n+2}(f)=S q_{w}^{\prime 2} f^{*}\left(\kappa_{n}\right)
$$

As in the proof of (14.3), to see (14.7) we may consider $K$ as a cellular complex composed of oriented open cells with $K_{n+2}$ written as in (14.4). We may assume also that $f$ is a cellular map. Define $f^{\prime}: X_{n+1} \rightarrow K$ by taking $f^{\prime}(x)$ $=C_{0}$ if $x \in X_{0}=f^{-1}\left(C_{n+1}^{s+1} \cup C_{n+2}^{s+2} \cup \cdots \cup C_{n+2}^{s}\right)$, and taking $f^{\prime}(x)=f(x)$ if $x \notin x_{0}$. Since $f^{-1}\left(C_{0}\right)$ separates $X_{n+1}, f^{\prime}$ is a map. Also, $f^{\prime}\left|X_{n}=f\right| X_{n}$, so $f^{\prime *}\left(\kappa_{n}\right)$ $=f^{*}\left(\kappa_{n}\right), Z^{n+2}\left(f^{\prime}\right)=Z^{n+2}(f)$, and $f^{\prime} \mid \partial \sigma_{n+2}$ represents an element of $\Gamma_{n+1}$ for any oriented $(n+2)$-simplex $\sigma_{n+2}$ in $X\left({ }^{17}\right)$.

(17) To see this we verify first that the boundary homomorphism: $\pi_{n+1}\left(K_{n} \cup C_{n+1}^{1} \cup \ldots\right.$ $\left.\cup C_{n+1}^{s}, K_{n}\right) \rightarrow \pi_{n}\left(K_{n}\right)$ is an into-isomorphism. 
By (14.6), $f^{\prime}$ is extendable to a map $F: X \rightarrow K * K$, and the commutativity in each square of the diagram

$$
\begin{array}{ccc}
H^{s}(K * K, K ; G) & \stackrel{\xi}{\rightarrow} H^{s}(K * K, G) \stackrel{\eta}{\rightarrow} H^{s}(K, G) \\
\downarrow & \downarrow F^{*} \quad \stackrel{\xi}{\xi} \quad c \quad \downarrow F^{*} \\
H^{s}\left(X, X_{n+1} ; G\right) & \stackrel{\xi}{\rightarrow} H^{s}(X, G) & \stackrel{\eta}{\rightarrow} H^{s}\left(X_{n_{+}+1} ; G\right)
\end{array}
$$

holds, where the upper homomorphisms $\xi$ and $\eta$ were given before, and the lower $\xi$ and $\eta$ are respectively the injection and projection homomorphisms in the cohomology sequence of $\left(X, X_{n+1}\right)$. Thus, since, by (14.6), for any oriented $(n+2)$-simplex $\sigma_{n+2}, \quad\left(F \mid \sigma_{n+2}\right)^{*}\left(u_{n+2}\right) \in H^{n+2}\left(\sigma_{n+2}, \partial \sigma_{n+2} ; \pi_{n+1}\right)$ is represented by $\left[f^{\prime} \mid \partial \sigma_{n+2}\right] \sigma_{n+2}, F^{*} S q_{w}^{\prime 2} \mu\left(K_{n}\right)=F^{*} \xi\left(u_{n+2}\right)=\xi F^{*}\left(u_{n+2}\right)=$ the element of $H^{n+2}\left(X, \pi_{n+1}\right)$ represented by $\sum_{\sigma_{n+2} \subset X}\left[f^{\prime} \mid \partial \sigma_{n+2}\right] \sigma_{n+2}$, namely, the secondary obstruction $Z^{n+2}\left(f^{\prime}\right)$. But, $F^{*} S q_{w}^{\prime 2} \mu\left(\kappa_{n}\right)=S q_{w}^{\prime 2} F^{*} \mu\left(\kappa_{n}\right)=S q_{w}^{\prime 2} f^{\prime *}\left(\kappa_{n}\right)$ (by (3.6)). This completes the proof of (14.7).

We may deduce in a similar manner as above and by arguments in $[16$, p. 78] the relative extension theorem on secondary obstructions.

\section{APPENDIX}

J. H. C. Whitehead, in Comment. Math. Helv. vol. 22 (1949) pp. 48-92 and Annales de la Société Polonaise de Mathématiques vol. 21 (1948) pp. 176186 , considered a particular type of $A_{n}^{2}$-complex, called a reduced $A_{n}^{2}$-complex, and showed that every $A_{n}^{2}$-complex is of the same homotopy type of a reduced one. This notion can be generalized. We give the exact formulation of this generalization in the following.

Consider a cellular complex $K$ composed of open $n$-cells $e_{n}^{i}$ 's with characteristic maps $f_{n i}: \sigma_{n} \rightarrow \bar{e}_{n}^{4}$ as given in [14, p. 221] satisfying the following condition $(\Delta)$, namely,

$(\Delta)$ For each $e_{n}^{i}$, in the boundary $\partial \sigma_{n}$, there are a finite number of non-overlapping cells $\left(^{8}\right)$, say $\tau_{n-1}^{1}, \tau_{n-1}^{2}, \cdots, \tau_{n-1}^{r}$, such that $(\alpha)$ for each $j$, we have a homeomorphism $g_{j}: \sigma_{n-1} \rightarrow \tau_{n-1}^{j}$ and $\left(f_{n i} \mid \tau_{n-1}^{j}\right) g_{j}$ is the characteristic map of some $e_{n-1}^{l}$, and that $(\beta) f_{n i}\left(\partial \sigma_{n}-\cup_{j-1}^{r} \tau_{n-1}^{\prime}\right) \subset K_{n-2}(=$ the $(n-2)$ th skeleton of $K)$.

For a given pair $\left(e_{n}^{i}, e_{n-1}^{l^{\prime}}\right)$, we denote by $\left[e_{n}^{i}, e_{n-1}^{i^{\prime}}\right]$ the total number of $\tau_{n-1}^{j}$ 's, say $\tau_{n-1}^{j_{1}}, \tau_{n-1}^{j_{2}}, \cdots, \tau_{n-1}^{j_{s}}$, such that $\left(f_{n i} \mid \tau_{n-1}^{\prime}\right) g_{j}$ is the characteristic map of $e_{n-1}^{t^{\prime}}$. ( $\left[e_{n}^{i}, e_{n-1}^{\prime}\right]$ may be zero.) Suppose that fixed orientations are assigned to the open cells of $K$. Let $\sigma_{n}, \tau_{n-1}^{j_{k}}$ be oriented coherently with $f_{n i}$, $\left(f_{n i} \mid \tau_{n-1}^{j_{k}}\right) g_{j_{k}}$ respectively. Then $\tau_{n-1}^{j_{k}}$ appears as a face in $\sigma_{n}$ positively or negatively, and we write $\left\{e_{n}^{i}, \tau_{n-1}^{j_{k}}\right\}=1$ or -1 according as the former or the latter is the case. We write $\left\{e_{n}^{i}, e_{n-1}^{i}\right\}=\sum_{k=1}^{s}\left\{e_{n}^{i}, \tau_{n-1}^{s_{k}}\right\}$. It may be called the incidence number of the pair $\left(e_{n}^{t}, e_{n-1}^{t}\right)$ and the homology boundary of $e_{n}^{t}$ will be given by $\partial e_{n}^{i}=\sum_{i^{\prime}}\left\{e_{n}^{i}, e_{n-1}^{i^{\prime}}\right\} e_{n-1}^{\prime^{\prime}}$.

We shall call a simply-connected finite cellular complex $K$ composed of oriented $n$-cells $e_{n}$ 's a reduced complex if it satisfies the condition $(\Delta)$ and 
satisfies also the following further conditions (i) and (ii):

(i) $\left[e_{n}, e_{n-1}\right]= \pm\left\{e_{n}, e_{n-1}\right\}$ for any pair $\left(e_{n}, e_{n-1}\right)$.

(ii) The $e_{n}^{\prime}$ 's can be numbered as

$$
e_{n}^{1}, e_{n}^{2}, \cdots, e_{n}^{i_{n}}, e_{n}^{i_{n}+1}, \cdots, e_{n}^{i_{n^{\prime}}}, e_{n}^{i_{n^{\prime}}+1}, \cdots, e_{n}^{i_{n^{\prime \prime}}}, \quad(n=0,1,2, \cdots),
$$

with $i_{0}^{\prime \prime}=1, i_{1}^{\prime}=0$ and $i_{n}^{\prime \prime}-i_{n}^{\prime}=i_{n-1}^{\prime}-i_{n-1}=j_{n}$ such that the homology boundary $\partial e_{n}^{i}=0$ for $1 \leqq i \leqq i_{n}^{\prime}{ }^{\prime}$ and $\partial e_{n}^{i^{\prime}{ }^{+1}}=\theta_{n-1, i} e_{n-1}^{i_{n-1}+i}$ with $\left|\theta_{n-1, i}\right| \geqq 2$ for $1 \leqq i \leqq j_{n}$.

Under these conditions, we see easily that the $n$th Betti number of $K$ is $i_{n}$, the $n$th torsion coefficients (with respect to homology) are $\theta_{n 1}, \theta_{n 2}, \cdots$, $\theta_{n j_{n+1}}$, and the homotopy boundary $\left({ }^{10}\right)$ of $e_{n}^{i}$ for $1 \leqq i \leqq i_{n}^{\prime}$ lies in $K_{n-2}$.

We have:

Every connected, simply-connected finite cellular complex is of the same homotopy type of some reduced complex.

This statement can be proved by some standard procedure in combinatorial homotopy theory. We wish to omit the detail here. The guiding idea in proving this may be the same as the reduction of incidence matrices of a finite complex into canonical form by elementary transformations $\left({ }^{18}\right)$. We may begin by taking the given cellular complex as simplicial [14, p. 239], so that it satisfies the condition $(\Delta)$. Besides, arguments such as follows will be useful. Let $K$ be an $n$-dimensional cellular complex which satisfies the condition $(\Delta)$ and whose secondary skeleton is the union of 2 -spheres contacting at one point. Then, $(\alpha)$ with $e_{n}^{i}, e_{n-1}^{i^{\prime}}, \tau_{n-1}^{\prime}$ 's given as before, if $\left\{e_{n}^{i}, \tau_{n-1}^{j^{\prime}}\right\}=1$, $\left\{e_{n}^{i}, \tau_{n-1}^{\tau^{\prime \prime}}\right\}=-1$, we may deform the homotopy boundary of $e_{n}^{i}$ without altering the homotopy type of $K$ such that the number $\left[e_{n}^{i}, e_{n-1}^{i^{\prime}}\right]$ is diminished by 2 and the resulting cellular complex still satisfies the condition $(\Delta)$. Next, $(\beta)$ if $\left[e_{n}^{i}, e_{n-1}^{i^{\prime}}\right]=1$ and $\left[e_{n}^{i^{\prime \prime}}, e_{n-1}^{t^{\prime}}\right]=0$ for all $e_{n}^{i^{\prime \prime}} \neq e_{n}^{i}$, the subcomplex $K-e_{n}^{i}$ $-e_{n-1}^{i^{\prime}}$ is a deformation retract of $K$.

\section{REFERENCES}

1. J. Adem, The iteration of the Steenrod squares in algebraic topology, Proc. Nat. Acad. Sci. U.S.A. vol. 38 (1952) pp. 720-726.

2. S. Eilenberg and S. MacLane, Cohomology theory of abelian groups and homotopy theory II, Proc. Nat. Acad. Sci. U.S.A. vol. 36 (1950) pp. 657-663.

3. S. D. Liao, A theorem on periodic transformations of homology sphere, Ann. of Math. vol. 56 (1952) pp. 68-83.

4. W. S. Massey and G. W. Whitehead, The $(n+3)$-dimensional homotopy group of the n-sphere, Bull. Amer. Math. Soc. vol. 57 (1951) pp. 491-492.

5. J. Moore, Applications of homology theory to homotopy problems, to appear in Ann. of Math. For abstracts, see Bull. Amer. Math. Soc. Abstracts 58-4-393-396; 58-6-721, 722.

6. J. P. Serre, Homologie singliere des espaces fibrés, Applications, Ann. of Math. vol. 54 (1951) pp. 452-505.

7. P. A. Smith, Periodic and nearly periodic transformations, Lectures on Topology, University of Michigan Press, 1941, pp. 159-190.

(18) H. Seifert and W. Threlfall, Lehrbuch der Topologie, Leipzig, Teubner, 1934, pp. 71-76. 
8. - Fixed points of periodic transformations, Appendix B in S. Lefschetz, Algebraic topology, Amer. Math. Soc. Colloquium Publications, vol. 27, New York, 1942.

9. N. E. Steenrod, Homology groups of symmetric groups and reduced power operations, Proc. Nat. Acad. Sci. U.S.A. vol. 39 (1953) pp. 213-217; Cyclic reduced powers of cohomology classes, Proc. Nat. Acad. Sci. U.S.A. vol. 39 (1953) pp. 217-223.

10. - Products of cocycles and extensions of mappings, Ann. of Math. vol. 48 (1947) pp. 290-320.

11. N. E. Steenrod and J. H. C. Whitehead, Vector fields on the $n$-sphere, Proc. Nat. Acad. Sci. U.S.A. vol. 37 (1951) pp. 58-63.

12. M. R. Thom, Une théorie intrinseque des puissances de Steenrod, Colloque de Topologie de Strasbourg, 1951 (mimeographed).

13. - Variêtés plongées et i-carrés, C. R. Acad. Sci. Paris vol. 230 (1950) pp. 508-511.

14. J. H. C. Whitehead, Combinatorial homotopy. I, Bull. Amer. Math. Soc. vol. 55 (1949) pp. 213-245.

15. - A certain exact sequence, Ann. of Math. vol. 52 (1950) pp. 51-110.

16. - On the theory of obstructions, Ann. of Math. vol. 54 (1951) pp. 68-84.

17. Wu Wen-Tsün, Sur les puissances de Steenrod, Colloques de Topologie de Strasbourg, 1951 (mimeographed).

The Institute for Advanced Study,

Princeton, N. J. 